\title{
Flavor decomposition of the pion-nucleon $\sigma$-term
}

\author{
Daniel Severt, ${ }^{a}$ Ulf-G. Meißner ${ }^{a, b, c}$ and Jambul Gegelia ${ }^{d, c}$ \\ ${ }^{a}$ Helmholtz-Institut für Strahlen- und Kernphysik and \\ Bethe Center for Theoretical Physics, Universität Bonn, \\ D-53115 Bonn, Germany \\ ${ }^{b}$ Institute for Advanced Simulation (IAS-4) Institut für Kernphysik (IKP-3) and JCHP, \\ Forschungszentrum Jülich, D-52425 Jülich, Germany \\ ${ }^{c}$ Tbilisi State University, \\ 0186 Tbilisi, Georgia \\ ${ }^{d}$ Fakultät für Physik und Astronomie, \\ Institut für Theoretische Physik II, Ruhr-Universität Bochum, \\ D-44870 Bochum, Germany \\ E-mail: severt@hiskp.uni-bonn.de, meissner@hiskp.uni-bonn.de, \\ jgegelia@hotmail.com
}

ABstract: We re-analyze the flavor decomposition of the pion-nucleon $\sigma$-term in the framework of baryon chiral perturbation to fourth order. We employ a covariant and the heavy baryon framework including also the low-lying decuplet. Using only continuum data, we find a small strangeness content of the proton. The uncertainties are, however, large and might be overcome by dedicated lattice QCD calculations.

KeYwords: Chiral Lagrangians, Effective Field Theories

ARXIV EPRINT: 1902.10508 


\section{Contents}

1 Short introduction $\quad 2$

2 Sigma term basics 2

2.1 Definitions 2

2.2 Method of calculation 3

2.3 Some phenomenology 4

3 Chiral Lagrangians $\quad 4$

3.1 Baryon Lagrangians 4

$\begin{array}{ll}3.2 & \text { The heavy-baryon approach }\end{array}$

3.3 The EOMS scheme $\quad 7$

$\begin{array}{ll}3.4 & \text { Inclusion of the decuplet baryon resonances }\end{array}$

3.4.1 Covariant Lagrangian $\quad 8$

3.4.2 HB approach for the decuplet $\quad 9$

3.5 Constraints on the LECs 9

4 Calculation of the baryon masses and the $\sigma$-term $\quad 10$

$\begin{array}{ll}4.1 \text { Second order } & 11\end{array}$

$\begin{array}{lll}4.2 \text { Third order } & 12\end{array}$

$\begin{array}{lll}4.3 & \text { Fourth order } & 13\end{array}$

$\begin{array}{lll}\text { 4.3.1 } & \text { Baryon masses } & 13\end{array}$

$\begin{array}{lll}4.3 .2 & \sigma \text {-term } & 17\end{array}$

$\begin{array}{llr}5 & \text { Fit procedure and error analysis } & 19\end{array}$

$\begin{array}{lll}5.1 & \text { Unconstrained fits } & 19\end{array}$

5.2 Constrained fits 21

$6 \quad$ Results and discussion $\quad 21$

$\begin{array}{lll}7 & \text { Conclusions and outlook } & 23\end{array}$

$\begin{array}{ll}\text { A Mesonic chiral Lagrangian } & 24\end{array}$

B Decuplet-baryon masses at $\mathcal{O}\left(p^{2}\right) \quad 25$

$\begin{array}{ll}\text { C Fit results } & 25\end{array}$ 


\section{Short introduction}

The pion-nucleon $\sigma$-term $\sigma_{\pi N}$ parameterizes the scalar couplings of the nucleon to the light up- and down-quarks. It also plays a key role in the search for physics beyond the Standard Model, such as direct-detection searches for dark matter, see e.g. [1-3], but also other searches that are sensitive to the scalar current coupling to nucleons, see e.g. $[4,5]$.

Of particular interest is its flavor decomposition, in which one rewrites the $\sigma$-term in terms of an $\mathrm{SU}(3)$ singlet $\sigma_{0}$ and the so-called strangeness fraction $y$ as $\sigma_{\pi N}=\sigma_{0} /(1-y)$. It is the quantity $\sigma_{0}$ that will be the central object of this study. In fact, as will be discussed later, since there is a discrepancy between dispersion theoretical and lattice QCD determinations of $\sigma_{\pi N}$, it is of interest to analyze $\sigma_{0}$ based on continuum data only. As we will see, the complete one-loop calculations of $\mathcal{O}\left(p^{4}\right)$ utilizing various formulations of baryon chiral perturbation theory (also including the decuplet) as done here allows one to pin down $\sigma_{0}$ more precisely than the already available leading one-loop $\mathcal{O}\left(p^{3}\right)$ calculations. The other novelty of our calculation is a better estimate of the theoretical uncertainty, not only due to the parameter variations within a given order but also due to the neglected higher orders. Not surprisingly, we find that the latter are quite sizeable at third order but much smaller than the errors within the order for the fourth order calculations.

Needless to say that the formalism developed here can also be applied to analyze the results of lattice QCD calculations at varying light and strange quark masses. Given the tension in the value of $\sigma_{\pi N}$ alluded to before, we refrain from doing that here. Ultimately, however, we believe that lattice QCD will allow for a more precise determination of the flavor decomposition of $\sigma_{\pi N}$.

The paper is organized as follows. We give the basic definitions concerning the pionnucleon $\sigma$-term, the method of calculation and a brief recapitulation of known results in section 2. In section 3 we present the chiral Lagrangians necessary for our calculation, and discuss constraints on various low-energy constants. Section 4 gives details on the calculations of the baryon masses and the sigma-term at second, third and fourth order, respectively. The fit procedure and error analysis methods are discussed in section 5 . The results and corresponding discussions are given in section 6 . We end with our conclusions in section 7. Various technicalities and formulas are relegated to the appendices.

\section{Sigma term basics}

\subsection{Definitions}

The pion-nucleon sigma-term $\sigma_{\pi N}$ is defined as the expectation value of the light flavor $(u, d)$ QCD quark mass term in the nucleon,

$$
\sigma_{\pi N}=\frac{\hat{m}}{2 m_{N}}\langle N|\bar{u} u+\bar{d} d| N\rangle,
$$

where $u$ and $d$ are the up- and down-quark fields, respectively, and $|N\rangle$ is a properly normalized nucleon state, i.e. $\langle N \mid N\rangle=1$, with mass $m_{N}=938.9 \mathrm{MeV}$. In what follows, we restrict ourselves to the isospin limit $m_{u}=m_{d}=\hat{m}$. This approximation is justified, 
because the masses of the $u$ and $d$ quark are very small compared to $\Lambda_{\mathrm{QCD}} \sim 250 \mathrm{MeV}$ and this assumption simplifies the calculations. There is also a sigma-term related to the strange quark field

$$
\sigma_{s}=\frac{m_{s}}{2 m_{N}}\langle N|\bar{s} s| N\rangle
$$

with the strange quark mass $m_{s}$. One can define another expression that characterizes the scalar nucleon structure, namely the strangeness content of the nucleon $y$. It is defined by

$$
y=\frac{2\langle N|\bar{s} s| N\rangle}{\langle N|\bar{u} u+\bar{d} d| N\rangle}=\frac{2 \hat{m}}{m_{s}} \frac{\sigma_{s}}{\sigma_{\pi N}} .
$$

In order to calculate the strangeness content, one usually rewrites the $\pi N$ sigma-term in the following way:

$$
\sigma_{\pi N}=\frac{\sigma_{0}}{1-y}
$$

where $\sigma_{0}$ is given by

$$
\sigma_{0}=\frac{\hat{m}}{2 m_{N}}\langle N|\bar{u} u+\bar{d} d-2 \bar{s} s| N\rangle .
$$

This $\sigma_{0}$ is the central quantity of the calculations in this paper, because it allows one to deduce the strangeneness content of the nucleon. For example, if $y$ is equal to zero, $\sigma_{\pi N}$ and $\sigma_{0}$ are identical and the nucleon has a pure $u$ - and $d$-quark content.

\subsection{Method of calculation}

A possible way to calculate $\sigma_{\pi N}$ is based on utilizing the Feynman-Hellmann theorem. The starting point is the trace of the energy-momentum tensor of QCD, which in the isospin limit reads (neglecting heavy quarks and higher order QCD corrections, for more details we refer e.g. to ref. [6])

$$
\left(T_{\mathrm{QCD}}\right)_{\mu}^{\mu}=\frac{\beta_{\mathrm{QCD}}}{2 g_{s}} F_{\mu \nu}^{a} F^{a, \mu \nu}+\hat{m}(\bar{u} u+\bar{d} d)+m_{s} \bar{s} s,
$$

with $\beta_{\mathrm{QCD}}$ the beta-function of QCD. The expectation value with a nucleon state follows as

$$
\left\langle N\left|\left(T_{\mathrm{QCD}}\right)_{\mu}{ }^{\mu}\right| N\right\rangle=\left\langle N\left|m_{N}^{2}\right| N\right\rangle=m_{N}^{2}\langle N \mid N\rangle=m_{N}^{2} .
$$

Further, the nucleon mass $m_{N}$ is a function of the quark masses $m_{N}\left(\hat{m}, m_{s}\right)$. Hence we can investigate eq. (2.7) by taking the derivative with respect to $\hat{m}$,

$$
\frac{\partial}{\partial \hat{m}}\left(m_{N}^{2}\right)=2 m_{N}\left(\frac{\partial m_{N}}{\partial \hat{m}}\right)=\left\langle N\left|\left(\frac{\partial}{\partial \hat{m}}\left(T_{\mathrm{QCD}}\right)_{\mu}{ }^{\mu}\right)\right| N\right\rangle+m_{N}^{2} \frac{\partial}{\partial \hat{m}}\langle N \mid N\rangle .
$$

The second term vanishes due to normalization and we are left with

$$
2 m_{N}\left(\frac{\partial m_{N}}{\partial \hat{m}}\right)=\left\langle N\left|\left(\frac{\partial}{\partial \hat{m}}\left(T_{\mathrm{QCD}}\right)_{\mu}^{\mu}\right)\right| N\right\rangle \stackrel{(2.6)}{=}\langle N|\bar{u} u+\bar{d} d| N\rangle .
$$

From this relation we see that

$$
\hat{m}\left(\frac{\partial m_{N}}{\partial \hat{m}}\right)=\frac{\hat{m}}{2 m_{N}}\langle N|\bar{u} u+\bar{d} d| N\rangle=\sigma_{\pi N},
$$


which is known as the Feynman-Hellmann theorem for the pion-nucleon sigma-term. A similar calculation, where the nucleon mass is differentiated with respect to the strange quark mass $m_{s}$, leads to $\sigma_{s}$,

$$
\sigma_{s}=m_{s}\left(\frac{\partial m_{N}}{\partial m_{s}}\right)
$$

Thus, the pion-nucleon sigma-term can be calculated if we know the nucleon mass as a function of the quark masses. In the continuum, the nucleon mass is calculable within chiral perturbation theory (CHPT). We will closely follow the procedure shown in ref. [7] to obtain the sigma-term and $\sigma_{0}$. Alternatively, one can utilize lattice QCD, but we will not follow that path here as explained in the introduction.

\subsection{Some phenomenology}

For a long time, the value of $\sigma_{\pi N}$ was taken as $(45 \pm 5) \mathrm{MeV}$ [8], but this value is now superseded by the recent Roy-Steiner analysis of pion-nucleon scattering that also includes the superb measurements from pionic hydrogen and deuterium, leading to $\sigma_{\pi N}=$ $(59.1 \pm 3.5) \mathrm{MeV}$ [9], for more details, see the review [10]. Even if one ignores the constraints from the pionic atom measurements, fitting a representation based on Roy-Steiner equations to the low-energy pion-nucleon scattering data base leads to a consistent but less precise value of $(58 \pm 5) \mathrm{MeV}$ [11].

So what do we know about $\sigma_{0}$ ? For a long time, the pioneering calculations of refs. [12] and [7] led to values of $(35 \pm 5) \mathrm{MeV}$ and $(36 \pm 7) \mathrm{MeV}$, respectively, taken together with the old value of $\sigma_{\pi N}$ suggesting a small strangeness content. This, however, clearly is at odds with the new value of $\sigma_{\pi N}$. However, more recent calculations in the heavy baryon as well as covariant scheme with and without delta contributions to third order gave a vary unclear picture, with central values of $\sigma_{0}$ ranging from 46 to $89 \mathrm{MeV}$, see table 3 of ref. [13], with disturbingly large differences between the HB and covariant approaches when the decuplet was included. Clearly, such a situation requires an improved fourth order calculation, as will be presented in the following.

\section{Chiral Lagrangians}

In what follows, we will utilize baryon chiral perturbation theory (BCHPT) in various formulations. Here, we briefly exhibit the pertinent chiral Lagrangians. Since our aim is the calculation of the octet baryon masses to obtain the sigma-terms up to chiral order $\mathcal{O}\left(p^{4}\right)$, we only consider the effective baryon Lagrangians, which are relevant for our calculations. As we seek the flavor decomposition of $\sigma_{\pi N}$, we must work in three-flavor baryon chiral perturbation theory. Note that the purely mesonic Lagrangian is given in appendix A together with some definitions of various basic quantities.

\subsection{Baryon Lagrangians}

The leading-order baryon Lagrangian with coupling to the octet-meson fields is given by, see e.g. [14],

$$
\mathcal{L}_{\phi B}^{(1)}=\operatorname{Tr}\left(\bar{B}\left(i \not D-m_{0}\right) B\right)+\frac{D}{2} \operatorname{Tr}\left(\bar{B} \gamma^{\mu} \gamma_{5}\left\{u_{\mu}, B\right\}\right)+\frac{F}{2} \operatorname{Tr}\left(\bar{B} \gamma^{\mu} \gamma_{5}\left[u_{\mu}, B\right]\right),
$$


where $m_{0}$ is the octet-baryon mass in the chiral limit and $B(x)$ is a traceless $3 \times 3$-matrix denoting the lowest-lying octet-baryon fields in flavor $\mathrm{SU}(3)$

$$
B(x)=\frac{1}{\sqrt{2}} \sum_{a=1}^{8} \lambda^{a} B^{a}(x)=\left(\begin{array}{ccc}
\frac{1}{\sqrt{2}} \Sigma^{0}+\frac{1}{\sqrt{6}} \Lambda & \Sigma^{+} & p \\
\Sigma^{-} & -\frac{1}{\sqrt{2}} \Sigma^{0}+\frac{1}{\sqrt{6}} \Lambda & n \\
\Xi^{-} & \Xi^{0} & -\frac{2}{\sqrt{6}} \Lambda
\end{array}\right) .
$$

The matrix $B$ transforms as $B \rightarrow K B K^{\dagger}$ under $\mathrm{SU}(3)_{L} \times \mathrm{SU}(3)_{R}$ transformations, where $K(L, R, U)$ is the so called compensator field. $K$ is an element of the conserved subgroup $\mathrm{SU}(3)_{V}$. It depends on the left- and right-handed fields $L, R$, and on the pseudo-Goldstone boson fields collected in $U(x)$, cf. appendix A, which makes it a local transformation. The covariant derivative is defined to obey the transformation property $D_{\mu} B \rightarrow K\left(D_{\mu} B\right) K^{\dagger}$ and is given by

$$
D_{\mu} B=\partial_{\mu} B+\left[\Gamma_{\mu}, B\right]
$$

with

$$
\Gamma_{\mu}=\frac{1}{2}\left\{u^{\dagger}\left(\partial_{\mu}-i r_{\mu}\right) u+u\left(\partial_{\mu}-i l_{\mu}\right) u^{\dagger}\right\},
$$

where $u=\sqrt{U}=\exp \left(i \phi /\left(2 F_{\phi}\right)\right)$. The chiral vielbein is given by

$$
u_{\mu}=i\left\{u^{\dagger}\left(\partial_{\mu}-i r_{\mu}\right) u-u\left(\partial_{\mu}-i l_{\mu}\right) u^{\dagger}\right\}
$$

which also transforms as $u_{\mu} \rightarrow K u_{\mu} K^{\dagger}$. As we are only interested in masses and $\sigma$ terms, we set the external fields $r_{\mu}$ and $l_{\mu}$ to zero. The expansion of the vielbein is $u_{\mu}=-\partial_{\mu} \phi / F_{\phi}+\mathcal{O}\left(\phi^{3}\right)$. Here, $\phi$ denotes the pseudoscalar fields (pseudo-Goldstone bosons) and $F_{\phi}$ is the decay constant (in the chiral limit). The second and third terms in eq. (3.1) introduce axial-vector interactions with the axial-vector coupling constants $D$ and $F$, which can be determined from semi-leptonic decays. Throughout, we use $D=0.80$ and $F=0.46$, so that $g_{A}=F+D=1.26$, with $g_{A}$ the nucleon axial-vector coupling. This is the first matching relation between the two- and three-flavor versions of BCHPT of relevance here.

From a power counting perspective the chiral vielbein contains derivatives of meson octet fields and counts as $\mathcal{O}(p)$. The octet-baryon mass term $m_{0}$ has chiral order zero, since it is of the same order of magnitude as the chiral symmetry breaking scale $\Lambda_{\chi}$ and thus cannot be used as a small expansion parameter. The same argument holds for the baryon momenta, which are generated by the derivative term $i \not D$. The difference $\left(i \not D-m_{0}\right)$, however, corresponds to $\left(\not p-m_{0}\right)$ in momentum space, which is considered to be small and therefore counts as $\mathcal{O}(p)$. These properties can be used to set up higher-order baryon Lagrangians.

The second order baryon Lagrangian includes terms with quark mass insertions that explicitly break the chiral symmetry, terms with two vielbeins $u_{\mu}$, and terms with external currents $[15,16]$, see also [17],

$$
\mathcal{L}_{\phi B}^{(2)}=\mathcal{L}_{\phi B}^{(2, s b .)}+\mathcal{L}_{\phi B}^{(2, i n t .)}
$$

where the explicit chiral symmetry breaking terms are given by

$$
\mathcal{L}_{\phi B}^{(2, s b .)}=b_{0} \operatorname{Tr}\left(\chi_{+}\right) \operatorname{Tr}(\bar{B} B)+b_{D} \operatorname{Tr}\left(\bar{B}\left\{\chi_{+}, B\right\}\right)+b_{F} \operatorname{Tr}\left(\bar{B}\left[\chi_{+}, B\right]\right),
$$


and the $\mathcal{O}\left(p^{2}\right)$ interaction terms by

$$
\begin{aligned}
\mathcal{L}_{\phi B}^{(2, \text { int. })}= & b_{1} \operatorname{Tr}\left(\bar{B}\left[u_{\mu},\left[u^{\mu}, B\right]\right]\right)+b_{2} \operatorname{Tr}\left(\bar{B}\left\{u_{\mu},\left\{u^{\mu}, B\right\}\right\}\right) \\
& +b_{3} \operatorname{Tr}\left(\bar{B}\left\{u_{\mu},\left[u^{\mu}, B\right]\right\}\right)+b_{4} \operatorname{Tr}(\bar{B} B) \operatorname{Tr}\left(u^{\mu} u_{\mu}\right) \\
& +i b_{5}\left(\operatorname{Tr}\left(\bar{B}\left[u^{\mu},\left[u^{\nu}, \gamma_{\mu} D_{\nu} B\right]\right]\right)-\operatorname{Tr}\left(\bar{B} \overleftarrow{D}_{\nu}\left[u^{\nu},\left[u^{\mu}, \gamma_{\mu} B\right]\right]\right)\right) \\
& +i b_{6}\left(\operatorname{Tr}\left(\bar{B}\left[u^{\mu},\left\{u^{\nu}, \gamma_{\mu} D_{\nu} B\right\}\right]\right)-\operatorname{Tr}\left(\bar{B} \overleftarrow{D}_{\nu}\left\{u^{\nu},\left[u^{\mu}, \gamma_{\mu} B\right]\right\}\right)\right) \\
& +i b_{7}\left(\operatorname{Tr}\left(\bar{B}\left\{u^{\mu},\left\{u^{\nu}, \gamma_{\mu} D_{\nu} B\right\}\right\}\right)-\operatorname{Tr}\left(\bar{B} \overleftarrow{D}_{\nu}\left\{u^{\nu},\left\{u^{\mu}, \gamma_{\mu} B\right\}\right\}\right)\right) \\
& +i b_{8}\left(\operatorname{Tr}\left(\bar{B} \gamma_{\mu} D_{\nu} B\right)-\operatorname{Tr}\left(\bar{B} \overleftarrow{D}_{\nu} \gamma_{\mu} B\right)\right) \operatorname{Tr}\left(u^{\mu} u^{\nu}\right)+\ldots,
\end{aligned}
$$

where $b_{0}, b_{D}, b_{F}$, and $b_{1,2, \ldots, 8}$ are LECs and $\chi_{+}=u^{\dagger} \chi u^{\dagger}+u \chi^{\dagger} u$. Note that $b_{0}, b_{D}, b_{F}$, and $b_{1, \ldots, 4}$ have dimension (mass) ${ }^{-1}$ and $b_{5, \ldots, 8}$ have (mass) $)^{-2}$. The ellipses in eq. (3.8) denotes terms that do not contribute to the calculation of the baryon masses up to order $\mathcal{O}\left(p^{4}\right)$. The third order meson-baryon Lagrangian does not contribute to the masses, because it generates meson-baryon interactions that will not enter before $\mathcal{O}\left(p^{5}\right)$. The fourth order Lagrangian, however, will contribute via tree level diagrams. The relevant part is given by

$$
\begin{aligned}
\mathcal{L}_{\phi B}^{(4)}= & d_{1} \operatorname{Tr}\left(\bar{B}\left[\chi_{+},\left[\chi_{+}, B\right]\right]\right)+d_{2} \operatorname{Tr}\left(\bar{B}\left[\chi_{+},\left\{\chi_{+}, B\right\}\right]\right) \\
& +d_{3} \operatorname{Tr}\left(\bar{B}\left\{\chi_{+},\left\{\chi_{+}, B\right\}\right\}\right)+d_{4} \operatorname{Tr}\left(\bar{B} \chi_{+}\right) \operatorname{Tr}\left(\chi_{+} B\right) \\
& +d_{5} \operatorname{Tr}\left(\bar{B}\left[\chi_{+}, B\right]\right) \operatorname{Tr}\left(\chi_{+}\right)+d_{7} \operatorname{Tr}(\bar{B} B)\left[\operatorname{Tr}\left(\chi_{+}\right)\right]^{2} \\
& +d_{8} \operatorname{Tr}(\bar{B} B) \operatorname{Tr}\left(\chi_{+}^{2}\right),
\end{aligned}
$$

where $d_{1, \ldots, 5}, d_{7}$, and $d_{8}$ are LECs as well with dimension (mass) ${ }^{-3}$.

\subsection{The heavy-baryon approach}

We have seen that the baryon Lagrangian at lowest order introduces a new parameter $m_{0}$, which is close to the chiral symmetry breaking scale and does not vanish in the chiral limit. It was first pointed out in [18] that this spoils the power counting scheme in loop calculations when using the relativistic nucleon propagator. In order to restore the power counting, the so-called heavy-baryon approach (HB) was introduced in ref. [19] and systematically developed in ref. [20]. The baryons are considered as very heavy sources with momentum

$$
p_{\mu}=m_{0} v_{\mu}+l_{\mu},
$$

where $v_{\mu}$ is the four-velocity subject to the constraint $v^{2}=1$, and $l_{\mu}$ is a small off-shell momentum, $v \cdot l \ll m_{0}$. The baryon field $B$ can then be written as

$$
B=\exp \left\{-i m_{0} v \cdot x\right\}\left(B_{v}+b_{v}\right),
$$

with a large component field $B_{v}$ and a small component field $b_{v}$, satisfying $\psi B_{v}=B_{v}$ and $\psi b_{v}=-b_{v}$. Inserting this into eq. (3.1) one obtains a Lagrangian in terms of $B_{v}$. Within the path integral formalism, one can shift the variable $b_{v}$ to absorb mixing terms of $b_{v}$ and $B_{v}$. Afterwards the $b_{v}$ field is integrated out. The result is given by

$$
\mathcal{L}_{H B}^{(1)}=\operatorname{Tr}\left(\bar{B}_{v}(i v \cdot D) B_{v}\right)+D \operatorname{Tr}\left(\bar{B}_{v} S^{\mu}\left\{u_{\mu}, B_{v}\right\}\right)+F \operatorname{Tr}\left(\bar{B}_{v} S^{\mu}\left[u_{\mu}, B_{v}\right]\right),
$$


plus terms of $\mathcal{O}\left(1 / m_{0}\right) . S^{\mu}$ is the covariant Pauli-Lubanski spin operator defined by $S^{\mu}=$ $-\gamma_{5}\left(\gamma^{\mu} \psi-v^{\mu}\right) / 2$, with $v \cdot S=0$ and $S^{2}=(1-D) / 4$ in $D$ space-time dimensions. The HB Lagrangian does not contain a mass term for $B_{v}$ and its corresponding propagator is

$$
S_{H B}^{a b}(\omega)=\frac{i \delta^{a b}}{v \cdot k+i \epsilon}, \quad \text { with } \omega=v \cdot k
$$

Using this heavy-baryon propagator in loop calculations restores the power counting, since the mass parameter $m_{0}$ does not appear. The problem with the HB approach, however, is that $m_{0}$ is not extremely large and in some calculations one expects significant corrections from $\mathcal{O}\left(1 / m_{0}\right)$ terms. For more details on this and also the representation of the effective Lagrangian in this basis, see the reviews [21, 22].

\subsection{The EOMS scheme}

Despite the fact that the heavy-baryon approach provides a good approximation for many calculations and restores the power counting, one was still interested to use the fully covariant meson-baryon Lagrangian. The idea was to use a different renormalization procedure, rather than the $\overline{\mathrm{MS}}$ and $\widetilde{\mathrm{MS}}$ schemes, which are commonly used in loop calculations. $\overline{\mathrm{MS}}$ and $\widetilde{M S}$ use redefinitions of the parameters in the Lagrangian to subtract the infinities, that arise from the loop diagrams. The $\widetilde{\mathrm{MS}}$ scheme is commonly used in CHPT calculations. The so-called extended-on-mass-shell (EOMS) renormalization scheme [23], which is nowadays often used in BCHPT calculations, achieves this. Within the EOMS scheme, one performs additional finite subtractions to cancel the power counting violating terms, i.e. the LECs in the Lagrangian absorb the infinities and the finite power counting breaking pieces. This ensures that a given diagram will not contribute to orders lower than its chiral dimension and therefore restores the power counting. The power counting violating terms can be found by expanding the loop functions in terms of quantities with a known chiral order, like $M_{\phi}(\phi=\pi, K, \eta),\left(\not p-m_{0}\right)$ or $\left(p^{2}-m_{0}^{2}\right)$. All terms with a lower chiral dimension than the diagram are then subtracted. Note that the proper matching of the EOMS scheme to the HB approach is discussed in [24]. We follow that paper in our work.

We will use the EOMS scheme (with $\widetilde{\mathrm{MS}}$ ) and the HB approach for the calculation of the baryon masses and compare the results. Explicit formulas will be given in the respective sections.

\subsection{Inclusion of the decuplet baryon resonances}

So far, we only considered Lagrangians describing the octet-baryon fields, the octet-meson fields and their interactions. However, it was argued early in [25] that the lowest-lying spin$3 / 2$ decuplet-baryon resonances can contribute significantly to the quantum corrections of the octet baryon observables. The average octet mass $\bar{m}_{B}$ and the average decuplet mass $\bar{m}_{D}$ are only separated by approximately $\bar{m}_{D}-\bar{m}_{B} \simeq 231 \mathrm{MeV}$ [26]. This separation is smaller than the $K$ or $\eta$ masses. Further, the coupling $g_{\pi N \Delta}$ between the delta-baryons, nucleons and pions is quite large, see e.g [27], so one expects notable effects from the decuplet. 


\subsubsection{Covariant Lagrangian}

The spin-3/2 decuplet-baryons are described by the Rarita-Schwinger fields $T_{\mu}^{a b c}$. We use the conventions from [21], where the $u, d$ and $s$ quarks are assigned the values 1,2 and 3, respectively, and $a, b, c \in\{1,2,3\}$. The decuplet fields are defined by

$$
\begin{aligned}
& T_{\mu}^{111}=\Delta_{\mu}^{++}, T_{\mu}^{112}=T_{\mu}^{121}=T_{\mu}^{211}=\frac{\Delta_{\mu}^{+}}{\sqrt{3}}, T_{\mu}^{122}=T_{\mu}^{212}=T_{\mu}^{221}=\frac{\Delta_{\mu}^{0}}{\sqrt{3}}, T_{\mu}^{222}=\Delta_{\mu}^{-}, \\
& T_{\mu}^{113}=T_{\mu}^{131}=T_{\mu}^{311}=\frac{\Sigma_{\mu}^{*+}}{\sqrt{3}}, T_{\mu}^{223}=T_{\mu}^{232}=T_{\mu}^{322}=\frac{\Sigma_{\mu}^{*-}}{\sqrt{3}} \\
& T_{\mu}^{123}=T_{\mu}^{132}=T_{\mu}^{213}=T_{\mu}^{231}=T_{\mu}^{312}=T_{\mu}^{321}=\frac{\Sigma_{\mu}^{* 0}}{\sqrt{6}} \\
& T_{\mu}^{133}=T_{\mu}^{313}=T_{\mu}^{331}=\frac{\Xi_{\mu}^{* 0}}{\sqrt{3}}, T_{\mu}^{233}=T_{\mu}^{323}=T_{\mu}^{332}=\frac{\Xi_{\mu}^{*-}}{\sqrt{3}}, T_{\mu}^{333}=\Omega_{\mu}^{-}
\end{aligned}
$$

$T_{\mu}^{a b c}$ is totally symmetric under permutations of $a, b$ and $c$. The first order decuplet Lagrangian reads

$$
\mathcal{L}_{D}^{(1)}=\bar{T}_{\mu}^{a b c}\left(i \gamma^{\mu \nu \rho} D_{\rho}-m_{D} \gamma^{\mu \nu}\right) T_{\nu, a b c},
$$

where $m_{D}$ is the decuplet-baryon mass in the chiral limit, $\gamma^{\mu \nu}:=\gamma^{\mu} \gamma^{\nu}-g^{\mu \nu}$, and $\gamma^{\mu \nu \rho}=$ $(1 / 2)\left\{\gamma^{\mu \nu}, \gamma^{\rho}\right\}$. The covariant derivative is of the same form as in the meson-baryon Lagrangian and given by

$$
D_{\rho} T_{\nu, a b c}:=\partial_{\rho} T_{\nu, a b c}+\left(\Gamma_{\rho}, T_{\nu}\right)_{a b c}
$$

with

$$
\left(\Gamma_{\rho}, T_{\nu}\right)_{a b c}:=\left(\Gamma_{\rho}\right)_{a}^{d} T_{\nu, d b c}+\left(\Gamma_{\rho}\right)_{b}^{d} T_{\nu, a d c}+\left(\Gamma_{\rho}\right)_{c}^{d} T_{\nu, a b d},
$$

where $\left(\Gamma_{\rho}\right)_{a}^{d}$ denotes the element in row $a$ and column $d$ of the chiral connection $\Gamma_{\rho}$. The relativistic spin-3/2 propagator in $D$ space-time dimensions takes the form

$$
G^{\rho \mu}(k)=\frac{-i\left(\not k+m_{D}\right)}{k^{2}-m_{D}^{2}+i \epsilon}\left(g^{\rho \mu}-\frac{1}{D-1} \gamma^{\rho} \gamma^{\mu}+\frac{k^{\rho} \gamma^{\mu}-\gamma^{\rho} k^{\mu}}{(D-1) m_{D}}-\frac{D-2}{(D-1) m_{D}^{2}} k^{\rho} k^{\mu}\right) .
$$

Due to the non-vanishing quark masses, there is also a second order symmetry breaking Lagrangian

$$
\mathcal{L}_{D}^{(2, s b .)}=\frac{t_{0}}{2} \operatorname{Tr}\left(\chi_{+}\right) \bar{T}_{\mu}^{a b c} g^{\mu \nu} T_{\nu, a b c}+\frac{t_{D}}{2} \bar{T}_{\mu}^{a b c} g^{\mu \nu}\left(\chi_{+}, T_{\nu}\right)_{a b c},
$$

with the LECs $t_{0}$ and $t_{D}$. The leading-order interaction between the decuplet fields, the octet baryons and the mesons of chiral order one is usually written as

$$
\mathcal{L}_{D B \phi}^{(1)}=\frac{\mathcal{C}}{2}\left\{\bar{T}_{\mu}^{a b c} \Theta^{\mu \nu}(z)\left(u_{\nu}\right)_{a}^{i} B_{b}^{j} \epsilon_{c i j}+\bar{B}_{j}^{b}\left(u_{\nu}\right)_{i}^{a} \Theta^{\nu \mu}(z) T_{\mu, a b c} \epsilon^{c i j}\right\},
$$

where $C$ is a coupling constant, which can for example be fixed from the decay $\Delta \rightarrow N \pi$, $\epsilon_{c i j}$ is the Levi-Civita tensor and

$$
\Theta^{\mu \nu}(z)=g^{\mu \nu}-\left(z+\frac{1}{2}\right) \gamma^{\mu} \gamma^{\nu}
$$


with the so-called off-shell parameter $z$ describing the coupling of the "off-shell" spin- $1 / 2$ components from the Rarita-Schwinger field. As shown in ref. [28], $z$ can be absorbed into redefinitions of certain LECs and is therefore redundant, see also ref. [29]. Nevertheless, we will use the value $z=1 / 2$ in our covariant calculations as done in ref. [30], see also ref. [31].

\subsubsection{HB approach for the decuplet}

Since the decuplet-baryons also possess a large mass, it is quite obvious to apply the heavybaryon approximation to them as well, for a detailed discussion see [32]. Analogous to the nucleon case, one expresses the decuplet fields in terms of velocity eigenstates,

$$
T_{\mu}^{a b c}=e^{-i m_{0} v \cdot x}\left\{\left(T_{v}\right)_{\mu}^{a b c}+\left(t_{v}\right)_{\mu}^{a b c}\right\} .
$$

Note that the exponential function contains the average octet-baryon mass in the chiral limit, $m_{0}$, and not the decuplet mass $m_{D}$. This is done to avoid complex exponential functions in the decuplet-octet-meson interaction term. The price we have to pay is that the mass parameter $m_{D}$ will not cancel completely in the Lagrangian. Instead, the HB Lagrangian maintains a mass scale $\Delta:=m_{D}-m_{0}$, which does not vanish in the chiral limit and counts as order $\mathcal{O}(p)$ within the power-counting scheme. It takes the form

$$
\begin{aligned}
\mathcal{L}_{D H B}^{(1)}= & -i \bar{T}_{\mu}^{a b c}(v \cdot D) T_{a b c}^{\mu}-\Delta \bar{T}_{\mu}^{a b c} T_{a b c}^{\mu} \\
& +\frac{\mathcal{C}}{2}\left\{\bar{T}_{\mu}^{a b c}\left(u^{\mu}\right)_{a}^{i} B_{b}^{j} \epsilon_{c i j}+\bar{B}_{j}^{b}\left(u_{\mu}\right)_{i}^{a} T_{a b c}^{\mu} \epsilon^{c i j}\right\} .
\end{aligned}
$$

In the HB approach the off-shell parameter is usually set to $-1 / 2$, so that $\Theta^{\mu \nu}$ reduces to the Minkowski metric $g^{\mu \nu}$. The propagator of the decuplet field (in $D$ dimensions) simplifies to

$$
G_{H B}^{\mu \nu}(k)=\frac{i P^{\mu \nu}}{v \cdot k-\Delta+i \epsilon}, \quad \text { with } P^{\mu \nu}=v^{\mu} v^{\nu}-g^{\mu \nu}-4\left(\frac{D-3}{D-1}\right) S^{\mu} S^{\nu},
$$

containing explicitly the scale $\Delta$. The projection operator $P^{\mu \nu}$ satisfies $v_{\mu} P^{\mu \nu}=P^{\mu \nu} v_{\nu}=0$ and $P_{\mu}^{\mu}=-2$.

\subsection{Constraints on the LECs}

From the above it is clear that the baryon masses are not sufficient to fix all the LECs that appear. In fact, this does not appear possible within the continuum. So one way would be to resort to lattice QCD, which allows to vary the quark masses and thus gives a better handle on the symmetry breaking LECs, see e.g. refs. [17, 33-40]. However, there is an unresolved discrepancy between the precise Roy-Steiner determination of $\sigma_{\pi N}$ and present lattice QCD calculations, see ref. [41] (and references therein). Therefore, we follow another path and try to constrain the LECs (or combinations thereof) as much as possible utilizing continuum data. We consider matching between the $\mathrm{SU}(3)$ and $\mathrm{SU}(2)$ versions of the effective field theory, which gives relations between the coupling constants. More precisely, integrating out the strange quark reduces three-flavor CHPT to the two-flavor theory. This program has been carried out in detail in refs. [42, 44]. We will use the 
matching relations given in eqs. $(5.4,5.5,5.6)$ of ref. [42] because these also include some information on the fourth order LECs $d_{i}$. The dimension-two SU(2) LECs $c_{1,2,3,4}$ have been most precisely determined from matching the Roy-Steiner analysis of pion-nucleon scattering to the CHPT amplitudes [45]. We use the values obtained in the standard power counting from that paper,

$$
c_{1}=-1.11(3), \quad c_{2}=3.13(3), \quad c_{3}=-5.61(6), \quad c_{4}=4.26(4),
$$

all in $\mathrm{GeV}^{-1}$. Note that we will not use the matching relation of $c_{4}$ as it involves dimensiontwo LECs that do not appear in the baryon masses, see ref. [44]. When the decuplet is included, the values of the $c_{i}$ are changed as the $\Delta(1232)$-contribution has to be subtracted. We follow ref. [27], adopting to the value of $g_{A}$ used here. The $\Delta(1232)$-contribution is given by

$$
c_{2}^{\Delta}=-c_{3}^{\Delta}=\frac{g_{A}^{2}\left(m_{\Delta}-m_{N}\right)}{2\left[\left(m_{\Delta}-m_{N}\right)^{2}-M_{\pi}^{2}\right]}=3.49 \mathrm{GeV}^{-1},
$$

with $m_{\Delta}$ and $m_{N}$ the average delta and nucleon mass, respectively. Note that the value of $c_{1}$ is assumed to be generated from scalar sources only [27]. The matching of the $c_{i}$ and other LECs between the deltafull and deltaless theory has been refined in [43]. Alternatively to constraining the $c_{i}$, one could match to the scattering length expressions given in [44], see also [46], but since the corresponding calculations are not available in the EOMS scheme to sufficient accuracy, we do not follow this path here.

\section{Calculation of the baryon masses and the $\sigma$-term}

Now we have all the information that we need to calculate the quantum corrections of the octet-baryon masses. These corrections are given by the baryon self-energy $\Sigma_{B}$, which can be determined from the one-particle-irreducible perturbative contribution to the two-point function of the baryon field $B$

$$
i S_{B}(\not p):=\frac{i}{\not p-m_{0}-\Sigma_{B}(\not p)} .
$$

The physical baryon mass $m_{B}$ is the pole of the propagator at $\not p=m_{B}$, i.e.

$$
m_{B}-m_{0}-\Sigma_{B}\left(\not p=m_{B}\right)=0 \quad \Rightarrow \quad m_{B}=m_{0}+\Sigma_{B}\left(\not p=m_{B}\right) .
$$

Since we do not have the exact form of $m_{B}$ and we only calculate the mass up to a specific order, we can only approximate the self-energy by setting $\not p=m_{0}+$ (h.o.c.). ${ }^{1}$ In the heavy baryon approach the propagator has a slightly different form, cf. eq. (3.13). The baryon mass is given by

$$
m_{B}=m_{0}+\Sigma_{H B}(\omega=0),
$$

where $\Sigma_{H B}$ is the self-energy in the HB approach and $\omega=v \cdot p$.

\footnotetext{
${ }^{1}$ Here, h.o.c. means higher-order corrections. We have to adjust the value of $\not p$ depending on the accuracy of our calculation.
} 


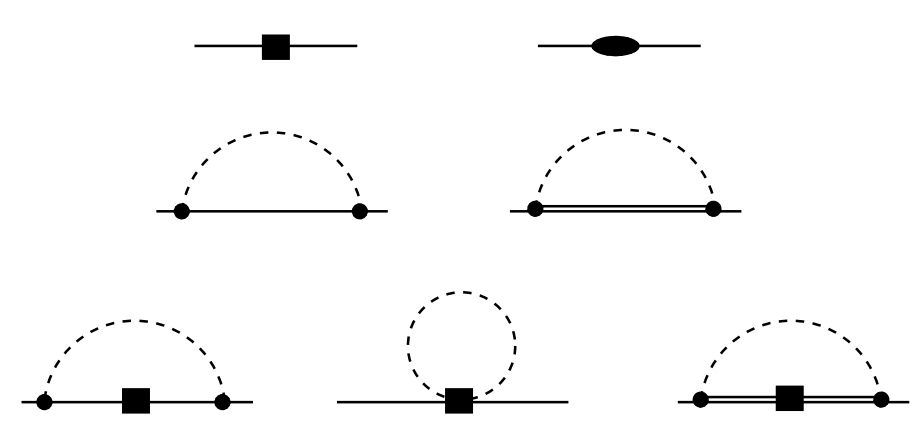

Figure 1. Feynman diagrams contributing to the masses up to fourth order. The contact terms at second and fourth order, the leading loop and the next-to-leading loop corrections are given in the first, second and third row, respectively. Solid, dashed, and solid double lines refer to octet baryons, Goldstone bosons, and decuplet states, respectively. Vertices denoted by a filled dot/square/ellipse refer to insertions from the first/second/fourth order chiral Lagrangian, respectively. Diagrams contributing via wave function renormalization only are not shown.

It is important to note that the self-energy - in the covariant as well as in the HB formulation - is a matrix $\Sigma^{b a}$, depending on the incoming baryon flavor index $a$ and the outgoing $b(a, b=1,2, \ldots, 8)$. The self-energies of the octet baryons $(N, \Sigma, \Lambda, \Xi)$ can be calculated by the following linear combinations [47]

$$
\Sigma_{N}=\Sigma^{44}-i \Sigma^{54}, \Sigma_{\Sigma}=\Sigma^{33}, \Sigma_{\Lambda}=\Sigma^{88}, \Sigma_{\Xi}=\Sigma^{44}+i \Sigma^{54}
$$

In order to calculate the masses/self-energies up to $\mathcal{O}\left(p^{4}\right)$, we need to consider all relevant terms of the effective Lagrangian

$$
\mathcal{L}_{\text {eff }}=\mathcal{L}_{\phi B}^{(1)}+\mathcal{L}_{\phi B}^{(2)}+\mathcal{L}_{\phi B}^{(4)}+\mathcal{L}_{\phi}^{(2)}+\mathcal{L}_{\phi}^{(4)}+\mathcal{L}_{D}^{(1)}+\mathcal{L}_{D}^{(2)}
$$

The various contributions to the self-energy at second, third and fourth order are depicted in figure 1 . The contributions from these orders will now be discussed separately.

\subsection{Second order}

The calculation of the tree graphs at $\mathcal{O}\left(p^{2}\right)$ is straightforward and well documented in the literature, cf. figure 1 (left diagram in the first row). The nucleon mass is given by (in both the HB and the EOMS scheme)

$$
m_{N}=m_{0}-\left(2 b_{0}+4 b_{F}\right) M_{\pi}^{2}-\left(4 b_{0}+4 b_{D}-4 b_{F}\right) M_{K}^{2},
$$

and the octet-baryon masses fulfill the Gell-Mann-Okubo relation,

$$
2\left(m_{N}+m_{\Xi}\right)=m_{\Sigma}+3 m_{\Lambda}
$$

which turns out to be approximately fulfilled in nature. For second and third order calculations, we use the mesonic Gell-Mann-Okubo relation

$$
3 M_{\eta}^{2}=4 M_{K}^{2}+M_{\pi}^{2},
$$


which is also approximately fulfilled in nature. For the sigma-term and its flavor singlet, we find

$$
\sigma_{\pi N}=-2 M_{\pi}^{2}\left(2 b_{0}+b_{D}+b_{F}\right), \quad \sigma_{0}=2 M_{\pi}^{2}\left(b_{D}-3 b_{F}\right) .
$$

As expected, the sigma-terms vanish in the chiral limit and they depend on the symmetrybreaking LECs. At this order, these LECs can be fixed from the baryon masses, leading to

$$
\sigma_{0}=\frac{1}{2}\left(\frac{M_{\pi}^{2}}{M_{K}^{2}-M_{\pi}^{2}}\right)\left(m_{\Xi}+m_{\Sigma}-2 m_{N}\right)
$$

Using the average masses for the corresponding meson and baryon isospin multiplets, one obtains

$$
\sigma_{0} \simeq 27 \mathrm{MeV}
$$

Taking $\sigma_{\pi N}=59.1 \mathrm{MeV}$ face value, this would lead to a strangeness content of $y \simeq 0.54$, which appears unacceptably large.

\subsection{Third order}

At third order, the baryon mass takes the generic form

$$
m_{B}=m_{0}+m_{B}^{(2)}+m_{B}^{(3)}+\delta m_{B}^{(3)}
$$

with $m_{B}^{(3)}$ the contribution from the leading one-loop diagrams with octet-baryon intermediate states and $\delta m_{B}^{(3)}$ the corresponding correction from the decuplet, cf. figure 1 (second row). As for the heavy baryon approach, the corresponding formulas can be found in ref. [26], with which we agree. For the theory without the decuplet, the EOMS expressions have been given first in ref. [47]. For the decuplet contribution within the EOMS scheme, we find (for a general value of the off-shell parameter $z$ and the renormalization scale set to $\mu=m_{D}$ ):

$$
\delta m_{B}^{(3)}=\frac{C^{2}}{48 \pi^{2} F_{\phi}^{2}}\left[\beta_{B \pi}^{(3)} \tilde{H}_{\mathrm{cov}}\left(M_{\pi}\right)+\beta_{B K}^{(3)} \tilde{H}_{\mathrm{cov}}\left(M_{K}\right)+\beta_{B \eta}^{(3)} \tilde{H}_{\mathrm{cov}}\left(M_{\eta}\right)\right],
$$

with

$$
\begin{aligned}
\tilde{H}_{\mathrm{cov}}\left(M_{\phi}\right)= & \frac{1}{96 m_{0}^{3} m_{D}^{2}}\left\{m _ { 0 } ^ { 2 } M _ { \phi } ^ { 2 } \left[6 m_{0}^{4}-2 m_{0}^{2}\left(6 m_{D}^{2}+M_{\phi}^{2}\left(5 z^{2}+4 z+2\right)\right)\right.\right. \\
& \left.+4 m_{0} m_{D} M_{\phi}^{2}\left(2 z^{2}-2 z-1\right)+3\left(2 m_{D}^{4}-3 m_{D}^{2} M_{\phi}^{2}+M_{\phi}^{4}\right)\right] \\
& -3 m_{D} M_{\phi}\left(m_{0}^{2}+2 m_{0} m_{D}+m_{D}^{2}-M_{\phi}^{2}\right)^{2}\left(M_{\phi}^{2}-\left(m_{0}-m_{D}\right)^{2}\right) \\
& \times \sqrt{\frac{-4 m_{D}^{2} M_{\phi}^{2}+\left(-m_{0}^{2}+m_{D}^{2}+M_{\phi}^{2}\right)^{2}}{m_{D}^{2} M_{\phi}^{2}}}
\end{aligned}
$$




$$
\begin{aligned}
& \times \log \left(\frac{-m_{0}^{2}+m_{D}^{2}+M_{\phi}^{2}}{2 m_{D} M_{\phi}}-\sqrt{\left(\frac{-m_{0}^{2}+m_{D}^{2}+M_{\phi}^{2}}{2 m_{D} M_{\phi}}\right)^{2}}-1\right) \\
& -3\left[-m_{0}^{8}-2 m_{0}^{7} m_{D}+2 m_{0} m_{D}\left(m_{D}^{2}-M_{\phi}^{2}\right)^{3}+\left(m_{D}^{2}-M_{\phi}^{2}\right)^{4}\right. \\
& +6 m_{0}^{5} m_{D}\left(m_{D}^{2}+M_{\phi}^{2}\right)+2 m_{0}^{6}\left(m_{D}^{2}+2 M_{\phi}^{2}\right)-2 m_{0}^{2}\left(m_{D}^{6}-3 m_{D}^{2} M_{\phi}^{4}+2 M_{\phi}^{6}\right) \\
& \left.+4 m_{0}^{4} M_{\phi}^{4}\left(2 z^{2}+4 z-1\right)-2 m_{0}^{3} m_{D}\left(3 m_{D}^{4}+M_{\phi}^{4}\left(-16 z^{2}-8 z+5\right)\right)\right] \\
& \left.\times \log \left(\frac{M_{\phi}}{m_{D}}\right)\right\}, \quad \text { for } M_{\phi}=M_{\pi} .
\end{aligned}
$$

and

$$
\begin{aligned}
\tilde{H}_{\mathrm{cov}}\left(M_{\phi}\right)= & \frac{1}{96 m_{0}^{3} m_{D}^{2}}\left\{m _ { 0 } ^ { 2 } M _ { \phi } ^ { 2 } \left[6 m_{0}^{4}-2 m_{0}^{2}\left(6 m_{D}^{2}+M_{\phi}^{2}\left(5 z^{2}+4 z+2\right)\right)\right.\right. \\
& \left.+4 m_{0} m_{D} M_{\phi}^{2}\left(2 z^{2}-2 z-1\right)+3\left(2 m_{D}^{4}-3 m_{D}^{2} M_{\phi}^{2}+M_{\phi}^{4}\right)\right] \\
& -3 m_{D} M_{\phi}\left(m_{0}^{2}+2 m_{0} m_{D}+m_{D}^{2}-M_{\phi}^{2}\right)^{2}\left(M_{\phi}^{2}-\left(m_{0}-m_{D}\right)^{2}\right) \\
& \times \sqrt{\frac{4 m_{D}^{2} M_{\phi}^{2}-\left(-m_{0}^{2}+m_{D}^{2}+M_{\phi}^{2}\right)^{2}}{m_{D}^{2} M_{\phi}^{2}} \arccos \left(\frac{-m_{0}^{2}+m_{D}^{2}+M_{\phi}^{2}}{2 m_{D} M_{\phi}}\right)} \\
& -3\left[-m_{0}^{8}-2 m_{0}^{7} m_{D}+2 m_{0} m_{D}\left(m_{D}^{2}-M_{\phi}^{2}\right)^{3}+\left(m_{D}^{2}-M_{\phi}^{2}\right)^{4}\right. \\
& +6 m_{0}^{5} m_{D}\left(m_{D}^{2}+M_{\phi}^{2}\right)+2 m_{0}^{6}\left(m_{D}^{2}+2 M_{\phi}^{2}\right)-2 m_{0}^{2}\left(m_{D}^{6}-3 m_{D}^{2} M_{\phi}^{4}+2 M_{\phi}^{6}\right) \\
& \left.+4 m_{0}^{4} M_{\phi}^{4}\left(2 z^{2}+4 z-1\right)-2 m_{0}^{3} m_{D}\left(3 m_{D}^{4}+M_{\phi}^{4}\left(-16 z^{2}-8 z+5\right)\right)\right] \\
& \left.\times \log \left(\frac{M_{\phi}}{m_{D}}\right)\right\}, \quad \text { for } M_{\phi}=M_{K}, M_{\eta},
\end{aligned}
$$

and the prefactors $\beta_{B \phi}^{(3)}$ are collected in table 1, see also [26]. The expressions within the curly brackets agree with the ones in ref. [30] for the specific choice $z=1 / 2$ taken there. Note, however, that some of the prefactors are mistyped in that reference.

\subsection{Fourth order}

\subsubsection{Baryon masses}

To start this section, we require the fourth order representation of the Goldstone boson masses. These have been given in the seminal paper [48] and will not be displayed here. 


\begin{tabular}{|c|c|c|c|c|}
\hline & $\mathrm{N}$ & $\Sigma$ & $\Lambda$ & $\Xi$ \\
\hline$\beta_{B \pi}^{(3)}$ & 8 & $\frac{4}{3}$ & 6 & 2 \\
\hline$\beta_{B K}^{(3)}$ & 2 & $\frac{20}{3}$ & 4 & 6 \\
\hline$\beta_{B \eta}^{(3)}$ & 0 & 2 & 0 & 2 \\
\hline
\end{tabular}

Table 1. Coefficients of the $\mathcal{O}\left(p^{3}\right)$ self-energy diagrams with the decuplet-baryon propagator.

\begin{tabular}{|c|c|c|c|c|}
\hline & $\mathrm{N}$ & $\Sigma$ & $\Lambda$ & $\Xi$ \\
\hline$\gamma_{B \pi}^{(4)}$ & $-4\left(4 d_{1}+2 d_{5}+d_{7}+3 d_{8}\right)$ & $-4\left(4 d_{3}+d_{7}+3 d_{8}\right)$ & $-4\left(4 d_{3}+\frac{8}{3} d_{4}+d_{7}+3 d_{8}\right)$ & $-4\left(4 d_{1}-2 d_{5}+d_{7}+3 d_{8}\right)$ \\
& & & & \\
\hline$\gamma_{B K}^{(4)}$ & $-16\left(d_{1}-d_{2}+d_{3}\right.$ & $-16\left(d_{7}+d_{8}\right)$ & $-16\left(\frac{8}{3} d_{3}+\frac{2}{3} d_{4}+d_{7}+d_{8}\right)$ & $-16\left(d_{1}+d_{2}+d_{3}\right.$ \\
& $\left.-d_{5}+d_{7}+d_{8}\right)$ & & & $\left.+d_{5}+d_{7}+d_{8}\right)$ \\
\hline$\gamma_{B \pi K}^{(4)}$ & $8\left(4 d_{1}-2 d_{2}-d_{5}\right.$ & $-16\left(d_{7}-d_{8}\right)$ & $16\left(\frac{8}{3} d_{3}+\frac{4}{3} d_{4}-d_{7}+d_{8}\right)$ & $8\left(4 d_{1}+2 d_{2}+d_{5}\right.$ \\
& $\left.-2 d_{7}+2 d_{8}\right)$ & & & $\left.-2 d_{7}+2 d_{8}\right)$ \\
\hline
\end{tabular}

Table 2. Coefficients of the $\mathcal{O}\left(p^{4}\right)$ contact interactions.

As concerns the baryon masses, we consider first the tree graphs at $\mathcal{O}\left(p^{4}\right)$, see the right diagram in the first row of figure 1 . Their contribution is readily evaluated as

$$
m_{B, c}^{(4)}=\gamma_{B \pi}^{(4)} M_{\pi}^{4}+\gamma_{B K}^{(4)} M_{K}^{4}+\gamma_{B \pi K}^{(4)} M_{\pi}^{2} M_{K}^{2},
$$

where the coefficients can be found in table 2. Note that this result is valid for the HB and the covariant calculation.

The tadpole diagrams can be expressed as

$$
m_{B, \text { tadpole }}^{(4)}=\left[m_{B}^{(4)}\right]^{[1]}+\left[m_{B}^{(4)}\right]^{[2]}+\left[m_{B}^{(4)}\right]^{[3]} .
$$

with

$$
\left[m_{B}^{(4)}\right]^{[1]}=\frac{1}{\left(4 \pi F_{\phi}\right)^{2}}\left\{\xi_{B \pi}^{[1]} M_{\pi}^{2} \log \left(\frac{M_{\pi}^{2}}{\mu^{2}}\right)+\xi_{B K}^{[1]} M_{K}^{2} \log \left(\frac{M_{K}^{2}}{\mu^{2}}\right) \xi_{B \eta}^{[1]} M_{\eta}^{2} \log \left(\frac{M_{\eta}^{2}}{\mu^{2}}\right)\right\},
$$

and

$$
\left[m_{B}^{(4)}\right]^{[2]}=\frac{1}{\left(4 \pi F_{\phi}\right)^{2}}\left\{\xi_{B \pi}^{[2]} M_{\pi}^{4} \log \left(\frac{M_{\pi}^{2}}{\mu^{2}}\right)+\xi_{B K}^{[2]} M_{K}^{4} \log \left(\frac{M_{K}^{2}}{\mu^{2}}\right)+\xi_{B \eta}^{[2]} M_{\eta}^{4} \log \left(\frac{M_{\eta}^{2}}{\mu^{2}}\right)\right\},
$$

and

$$
\begin{gathered}
{\left[m_{B}^{(4)}\right]^{[3]}=\frac{m_{0}}{\left(4 \pi F_{\phi}\right)^{2}}\left\{\xi_{B \pi}^{[3]}\left[\frac{M_{\pi}^{4}}{4} \log \left(\frac{M_{\pi}^{2}}{\mu^{2}}\right)-\frac{M_{\pi}^{4}}{8}\right]+\xi_{B K}^{[3]}\left[\frac{M_{K}^{4}}{4} \log \left(\frac{M_{K}^{2}}{\mu^{2}}\right)-\frac{M_{K}^{4}}{8}\right]\right.} \\
\left.+\xi_{B \eta}^{[3]}\left[\frac{M_{\eta}^{4}}{4} \log \left(\frac{M_{\eta}^{2}}{\mu^{2}}\right)-\frac{M_{\eta}^{4}}{8}\right]\right\},
\end{gathered}
$$

and the various coefficients are collected in table 3 . Together with the contact interac- 


\begin{tabular}{|c|c|c|c|c|}
\hline$\xi_{B \pi}^{[1]}$ & $3\left(2 b_{0}+b_{D}+b_{F}\right) M_{\pi}^{2}$ & $6\left(b_{0}+b_{D}\right) M_{\pi}^{2}$ & $2\left(3 b_{0}+b_{D}\right) M_{\pi}^{2}$ & $3\left(2 b_{0}+b_{D}-b_{F}\right) M_{\pi}^{2}$ \\
\hline$\xi_{B K}^{[1]}$ & $2\left(4 b_{0}+3 b_{D}-b_{F}\right) M_{K}^{2}$ & $4\left(2 b_{0}+b_{D}\right) M_{K}^{2}$ & $\frac{4}{3}\left(6 b_{0}+5 b_{D}\right) M_{K}^{2}$ & $2\left(4 b_{0}+3 b_{D}+b_{F}\right) M_{K}^{2}$ \\
\hline$\xi_{B \eta}^{[1]}$ & $\frac{1}{3}\left[8\left(b_{0}+b_{D}-b_{F}\right) M_{K}^{2}\right.$ & $\frac{2}{3}\left[4 b_{0} M_{K}^{2}\right.$ & $\frac{2}{9}\left[4\left(3 b_{0}+4 b_{D}\right) M_{K}^{2}\right.$ & $\frac{1}{3}\left[8\left(b_{0}+b_{D}+b_{F}\right) M_{K}^{2}\right.$ \\
\hline$\xi_{B \pi}^{[2]}$ & $\left.-\left(2 b_{0}+3 b_{D}-5 b_{F}\right) M_{\pi}^{2}\right]$ & $\left.+\left(b_{D}-b_{F}\right) M_{\pi}^{2}\right]$ & $\left.-\left(3 b_{0}+7 b_{D}\right) M_{\pi}^{2}\right]$ & $\left.-\left(2 b_{0}+3 b_{D}+5 b_{F}\right) M_{\pi}^{2}\right]$ \\
\hline$\xi_{B K}^{[2]}$ & $-2\left(3 b_{1}+b_{2}+b_{3}+2 b_{4}\right)$ & $-2\left(4 b_{1}+2 b_{2}+3 b_{4}\right)$ & $-2\left(2 b_{2}+3 b_{4}\right)$ & $-3\left(b_{1}+b_{2}-b_{3}+2 b_{4}\right)$ \\
\hline$\left.\xi_{B \eta}^{[2]}+4 b_{4}\right)$ & $-4\left(b_{1}+b_{2}+2 b_{4}\right)$ & $-\frac{4}{3}\left(9 b_{1}+b_{2}+6 b_{4}\right)$ & $-2\left(3 b_{1}+3 b_{2}+b_{3}+4 b_{4}\right)$ \\
\hline$\xi_{B \pi}^{[3]}$ & $-6\left(b_{5}+b_{6}+b_{7}+2 b_{8}\right)$ & $-4\left(4 b_{5}+2 b_{7}+3 b_{8}\right)$ & $-4\left(2 b_{7}+3 b_{8}\right)$ & $-6\left(b_{5}-b_{6}+b_{7}+2 b_{8}\right)$ \\
\hline$\xi_{B K}^{[3]}$ & $-4\left(3 b_{5}+b_{6}+3 b_{7}+4 b_{8}\right)$ & $-8\left(b_{5}+b_{7}+2 b_{8}\right)$ & $-\frac{8}{3}\left(9 b_{5}+b_{7}+6 b_{8}\right)$ & $-4\left(3 b_{5}+b_{6}+3 b_{7}+4 b_{8}\right)$ \\
\hline$\xi_{B \eta}^{[3]}$ & $-\frac{2}{3}\left(9 b_{5}-3 b_{6}+b_{7}+6 b_{8}\right)$ & $-\frac{4}{3}\left(2 b_{7}+3 b_{8}\right)$ & $-4\left(2 b_{7}+b_{8}\right)$ & $-\frac{2}{3}\left(9 b_{5}+3 b_{6}+b_{7}+6 b_{8}\right)$ \\
\hline
\end{tabular}

Table 3. Coefficients of the $\mathcal{O}\left(p^{4}\right)$ tadpole diagrams.

\begin{tabular}{|c|c|c|c|c|}
\hline & $\mathrm{N}$ & $\Sigma$ & $\Lambda$ & $\Xi$ \\
\hline$\alpha_{B \pi}^{(4)}$ & $-\frac{3}{4}(D+F)^{2} m_{N}^{(2)}$ & $-\frac{1}{3} D^{2} m_{\Lambda}^{(2)}-2 F^{2} m_{\Sigma}^{(2)}$ & $-D^{2} m_{\Sigma}^{(2)}$ & $-\frac{3}{4}(D-F)^{2} m_{\Xi}^{(2)}$ \\
& & & & \\
\hline$\alpha_{B K}^{(4)}$ & $-\frac{1}{12}(D+3 F)^{2} m_{\Lambda}^{(2)}$ & $-\frac{1}{2}\left(D^{2}+F^{2}\right)\left(m_{N}^{(2)}+m_{\Xi}^{(2)}\right)$ & $-\frac{1}{6}\left(D^{2}+9 F^{2}\right)\left(m_{N}^{(2)}+m_{\Xi}^{(2)}\right)$ & $-\frac{1}{12}(D-3 F)^{2} m_{\Lambda}^{(2)}$ \\
& $-\frac{3}{4}(D-F)^{2} m_{\Sigma}^{(2)}$ & $+D F\left(m_{N}^{(2)}-m_{\Xi}^{(2)}\right)$ & $-D F\left(m_{N}^{(2)}-m_{\Xi}^{(2)}\right)$ & $-\frac{3}{4}(D+F)^{2} m_{\Sigma}^{(2)}$ \\
\hline$\alpha_{B \eta}^{(4)}$ & $-\frac{1}{12}(D-3 F)^{2} m_{N}^{(2)}$ & $-\frac{1}{3} D^{2} m_{\Sigma}^{(2)}$ & $-\frac{1}{3} D^{2} m_{\Lambda}^{(2)}$ & $-\frac{1}{12}(D+3 F)^{2} m_{\Xi}^{(2)}$ \\
& & & & \\
\hline
\end{tabular}

Table 4. Coefficients of the $\mathcal{O}\left(p^{4}\right)$ self-energy diagram.

tions from eq. (4.16) there are 15 LECs from the meson-baryon Lagrangian entering the calculation.

The next contribution comes from the loop diagram with two baryon propagators. First, we recall the HB result, see also refs. [7, 42]. The $\mathcal{O}\left(p^{4}\right)$ mass contribution in HB is given by

$$
\begin{aligned}
m_{B, H B \text { loop }}^{(4)}=-\frac{1}{\left(4 \pi F_{\phi}\right)^{2}}[ & \alpha_{B \pi}^{(4)} M_{\pi}^{2}\left(2+3 \log \left(\frac{M_{\pi}^{2}}{\mu^{2}}\right)\right)+\alpha_{B K}^{(4)} M_{K}^{2}\left(2+3 \log \left(\frac{M_{K}^{2}}{\mu^{2}}\right)\right) \\
& \left.+\alpha_{B \eta}^{(4)} M_{\eta}^{2}\left(2+3 \log \left(\frac{M_{\eta}^{2}}{\mu^{2}}\right)\right)\right]
\end{aligned}
$$

with the coefficients from table 4. Now we turn to the EOMS scheme. After some algebra, 


\begin{tabular}{|l|l|l|l|}
\hline $\mathrm{N}$ & $\Sigma$ & $\Lambda$ & $\Xi$ \\
\hline$\alpha_{N N \pi}^{(4)}=\frac{3}{4}(D+F)^{2}$ & $\alpha_{\Sigma \Sigma \pi}^{(4)}=2 F^{2}$ & & $\alpha_{\Xi \Xi \pi}^{(4)}=\frac{3}{4}(D-F)^{2}$ \\
\hline$\alpha_{N N \eta}^{(4)}=\frac{1}{12}(D-3 F)^{2}$ & $\alpha_{\Sigma \Sigma \eta}^{(4)}=\frac{1}{3} D^{2}$ & $\alpha_{\Lambda \Lambda \eta}^{(4)}=\frac{1}{3} D^{2}$ & $\alpha_{\Xi \Xi \eta}^{(4)}=\frac{1}{12}(D+3 F)^{2}$ \\
\hline$\alpha_{N \Lambda K}^{(4)}=\frac{1}{12}(D+3 F)^{2}$ & $\alpha_{\Sigma \Lambda \pi}^{(4)}=\frac{1}{3} D^{2}$ & $\alpha_{\Lambda \Sigma \pi}^{(4)}=D^{2}$ & $\alpha_{\Xi \Lambda K}^{(4)}=\frac{1}{12}(D-3 F)^{2}$ \\
\hline$\alpha_{N \Sigma K}^{(4)}=\frac{3}{4}(D-F)^{2}$ & $\alpha_{\Sigma N K}^{(4)}=\frac{1}{2}(D-F)^{2}$ & $\alpha_{\Lambda N K}^{(4)}=\frac{1}{6}(D+3 F)^{2}$ & $\alpha_{\Xi \Sigma K}^{(4)}=\frac{3}{4}(D+F)^{2}$ \\
\hline & $\alpha_{\Sigma \Xi K}^{(4)}=\frac{1}{2}(D+F)^{2}$ & $\alpha_{\Lambda \Xi K}^{(4)}=\frac{1}{6}(D-3 F)^{2}$ & \\
\hline
\end{tabular}

Table 5. Combined coefficients of the $\mathcal{O}\left(p^{4}\right)$ self-energy diagram.

one can compactly express the fourth order loop corrections as

$$
m_{B, \text { loop }}^{(4)}=\frac{1}{\left(4 \pi F_{\phi}\right)^{2}} \sum_{\phi} \sum_{B^{\prime}} \alpha_{B B^{\prime} \phi}^{(4)} \mathcal{E}_{B^{\prime} B}^{(4)}\left(M_{\phi}\right)
$$

where $B^{\prime} \in\{N, \Sigma, \Lambda, \Xi\}, \phi \in\{\pi, K, \eta\}$ and

$$
\begin{gathered}
\mathcal{E}_{B^{\prime} B}^{(4)}\left(M_{\phi}\right)=\frac{2 M_{\phi}^{3}}{m_{0}^{2} \sqrt{4 m_{0}^{2}-M_{\phi}^{2}}}\left[6 m_{0}^{2}\left(m_{B}^{(2)}-m_{B^{\prime}}^{(2)}\right)-M_{\phi}^{2}\left(2 m_{B}^{(2)}-m_{B^{\prime}}^{(2)}\right)\right] \arccos \left(\frac{M_{\phi}}{2 m_{0}}\right) \\
-M_{\phi}^{2}\left[2\left(2 m_{B}^{(2)}-m_{B^{\prime}}^{(2)}\right)+\left(m_{B}^{(2)}+m_{B^{\prime}}^{(2)}\right) \log \left(\frac{m_{0}^{2}}{\mu^{2}}\right)\right. \\
\left.+\frac{3 m_{0}^{2}\left(m_{B}^{(2)}-m_{B^{\prime}}^{(2)}\right)-M_{\phi}^{2}\left(2 m_{B}^{(2)}-m_{B^{\prime}}^{(2)}\right)}{m_{0}^{2}} \log \left(\frac{M_{\phi}^{2}}{m_{0}^{2}}\right)\right] .
\end{gathered}
$$

The coefficients $\alpha_{B B^{\prime} \phi}^{(4)}$ are given in table 5 .

Finally, we must consider the decuplet contribution at this order, see the rightmost diagram in the third row of figure 1. In the HB approach, the fourth order baryon mass shift takes the form

$$
\delta m_{B, H B}^{(4)}=\frac{\mathcal{C}^{2}}{64 \pi^{2} F_{\phi}^{2}}\left\{\beta_{B \pi}^{(4)} H^{(4)}\left(M_{\pi}\right)+\beta_{B K}^{(4)} H^{(4)}\left(M_{K}\right)+\beta_{B \eta}^{(4)} H^{(4)}\left(M_{\eta}\right)\right\}
$$

with

$$
H^{(4)}\left(M_{\phi}\right)=\left(4 \Delta^{2}-2 M_{\phi}^{2}\right) \log \left(\frac{M_{\phi}}{\mu}\right)-M_{\phi}^{2}-4 \Delta \sqrt{\Delta^{2}-M_{\phi}^{2}} \log \left(\frac{\Delta}{M_{\phi}}+\sqrt{\frac{\Delta^{2}}{M_{\phi}^{2}}-1}\right),
$$




\begin{tabular}{|c|c|c|c|c|}
\hline & $\mathrm{N}$ & $\Sigma$ & $\Lambda$ & $\Xi$ \\
\hline$\beta_{B \pi}^{(4)}$ & $8 m_{\Delta}^{(2)}$ & $\frac{4}{3} m_{\Sigma^{*}}^{(2)}$ & $6 m_{\Sigma^{*}}^{(2)}$ & $2 m_{\Xi^{*}}^{(2)}$ \\
\hline$\beta_{B K}^{(4)}$ & $2 m_{\Sigma^{*}}^{(2)}$ & $\frac{4}{3}\left[m_{\Xi^{*}}^{(2)}+4 m_{\Delta}^{(2)}\right]$ & $4 m_{\Xi^{*}}^{(2)}$ & $2\left[2 m_{\Omega^{-}}+m_{\Sigma^{*}}^{(2)}\right]$ \\
\hline$\beta_{B \eta}^{(4)}$ & 0 & $2 m_{\Sigma^{*}}^{(2)}$ & 0 & $2 m_{\Xi^{*}}^{(2)}$ \\
\hline
\end{tabular}

Table 6. Coefficients of the $\mathcal{O}\left(p^{4}\right)$ decuplet self-energy diagram.

for $M_{\phi}=M_{\pi}$ and

$$
H^{(4)}\left(M_{\phi}\right)=\left(4 \Delta^{2}-2 M_{\phi}^{2}\right) \log \left(\frac{M_{\phi}}{\mu}\right)-M_{\phi}^{2}-4 \Delta \sqrt{M_{\phi}^{2}-\Delta^{2}} \arccos \left(\frac{\Delta}{M_{\phi}}\right),
$$

for $M_{\phi}=M_{K}, M_{\eta}$, and the corresponding coefficients are given in table 6 . The covariant calculation is somewhat lengthy, we only give a short representation of the final results. There are indeed two contributions, one form the genuine $\mathcal{O}\left(p^{4}\right)$ diagram just discussed in the HB approach and the other one from the leading covariant $\mathcal{O}\left(p^{3}\right)$ diagram, that also generates a fourth order correction. The first contribution takes the from

$$
\delta m_{B, 1}^{(4)}=\frac{\mathcal{C}^{2}}{13824 \pi^{2} F_{\phi}^{2}}\left\{\beta_{B \pi}^{(4)} \tilde{H}_{\mathrm{cov}}^{(4), R}\left(M_{\pi}\right)+\beta_{B K}^{(4)} \tilde{H}_{\mathrm{cov}}^{(4), R}\left(M_{K}\right)+\beta_{B \eta}^{(4)} \tilde{H}_{\mathrm{cov}}^{(4), R}\left(M_{\eta}\right)\right\},
$$

with the coefficients from table 4 and the function $\tilde{H}_{\text {cov }}^{(4), R}\left(M_{\phi}\right)$ can be found in ref. [49]. The $\mathcal{O}\left(p^{4}\right)$ contribution from the $\mathcal{O}\left(p^{3}\right)$ diagram is proportional to the second order octetbaryon masses. It reads

$$
\delta m_{B, 2}^{(4)}=\frac{m_{B}^{(2)} \mathcal{C}^{2}}{9216 \pi^{2} F_{\phi}^{2}}\left\{\beta_{B \pi}^{(3)} \tilde{H}_{\mathrm{cov}}^{(3,4), R}\left(M_{\pi}\right)+\beta_{B K}^{(3)} \tilde{H}_{\mathrm{cov}}^{(3,4), R}\left(M_{K}\right)+\beta_{B \eta}^{(3)} \tilde{H}_{\mathrm{cov}}^{(3,4), R}\left(M_{\eta}\right)\right\}
$$

with the coefficients from table 1 and the function $\tilde{H}_{\text {cov }}^{(3,4), R}\left(M_{\phi}\right)$ is also given in ref. [49].

We have thus completed the calculation of all the self-energies contributing to the octet-baryon mass at chiral order $\mathcal{O}\left(p^{4}\right)$. Overall we have 22 new LECs at fourth order, with 15 coming from the $\mathcal{O}\left(p^{2}\right)$ and $\mathcal{O}\left(p^{4}\right)$ meson-baryon Lagrangians, 5 from the $\mathcal{O}\left(p^{4}\right)$ meson Lagrangian and 2 from the $\mathcal{O}\left(p^{2}\right)$ decuplet Lagrangian.

\subsection{2 $\sigma$-term}

Finally, we consider the sigma-term. It is calculated by using

$$
\frac{\partial m_{N}}{\partial \hat{m}}=\left(\frac{\partial m_{N}}{\partial M_{\pi}}\right) \frac{\partial M_{\pi}}{\partial \hat{m}}+\left(\frac{\partial m_{N}}{\partial M_{K}}\right) \frac{\partial M_{K}}{\partial \hat{m}}+\left(\frac{\partial m_{N}}{\partial M_{\eta}}\right) \frac{\partial M_{\eta}}{\partial \hat{m}} .
$$

This requires the Goldstone boson masses at fourth order to calculate the derivatives of the meson masses with respect to the quark masses $\hat{m}$ and $m_{s}$. Putting pieces together, 
we obtain for $\sigma_{\pi N}$ and $\sigma_{0}$ at fourth order

$$
\begin{aligned}
& \sigma_{\pi N}^{(4)}=\frac{1}{2 M_{\pi}}\left\{M_{\pi}^{2}+\frac{16 M_{\pi}^{4}}{F_{\phi}^{2}}\left[\left(2 L_{6}^{r}-L_{4}^{r}\right)+\frac{1}{2}\left(2 L_{8}^{r}-L_{5}^{r}\right)\right]+\frac{M_{\pi}^{4}}{36 \pi^{2} F_{\phi}^{2}}\right. \\
& \left.+\frac{M_{\pi}^{4}}{32 \pi^{2} F_{\phi}^{2}} \log \left(\frac{M_{\pi}^{2}}{\mu^{2}}\right)-\frac{M_{\pi}^{4}}{288 \pi^{2} F_{\phi}^{2}} \log \left(\frac{M_{\eta}^{2}}{\mu^{2}}\right)\right\} \cdot\left(\frac{\partial m_{N}}{\partial M_{\pi}}\right) \\
& +\frac{1}{2 M_{K}}\left\{\frac{M_{\pi}^{2}}{2}+\frac{4 M_{\pi}^{4}}{F_{\phi}^{2}}\left[4\left(2 L_{6}^{r}-L_{4}^{r}\right) M_{K}^{2}+\left(2 L_{8}^{r}-L_{5}^{r}\right)\left(2 M_{K}^{2}-M_{\pi}^{2}\right)\right]+\frac{M_{K}^{2} M_{\pi}^{2}}{144 \pi^{2} F_{\phi}^{2}}\right. \\
& \left.+\left[\frac{M_{\eta}^{2} M_{\pi}^{2}}{64 \pi^{2} F_{\phi}^{2}}+\frac{M_{K}^{2} M_{\pi}^{2}}{144 \pi^{2} F_{\phi}^{2}}\right] \log \left(\frac{M_{\eta}^{2}}{\mu^{2}}\right)-\frac{M_{\pi}^{4}}{64 \pi^{2} F_{\phi}^{2}} \log \left(\frac{M_{\pi}^{2}}{\mu^{2}}\right)\right\} \cdot\left(\frac{\partial m_{N}}{\partial M_{K}}\right) \\
& +\frac{1}{2 M_{\eta}}\left\{\frac { M _ { \pi } ^ { 2 } } { 2 8 8 \pi ^ { 2 } F _ { \phi } ^ { 2 } } \left[96 \pi^{2} F_{\phi}^{2}+4608 \pi^{2}\left(2 L_{6}^{r}-L_{4}^{r}\right) M_{\eta}^{2}-4096 \pi^{2}\left(3 L_{7}^{r}+L_{8}^{r}\right) M_{K}^{2}\right.\right. \\
& +4096 \pi^{2}\left(3 L_{7}^{r}+L_{8}^{r}\right) M_{\pi}^{2}+1536 \pi^{2}\left(2 L_{8}^{r}-L_{5}^{r}\right) M_{\eta}^{2}-768 \pi^{2}\left(2 L_{8}^{r}-L_{5}^{r}\right) M_{\pi}^{2} \\
& \left.+5 M_{\eta}^{2}-5 M_{\pi}^{2}\right]+M_{\pi}^{2} \log \left(\frac{M_{\eta}^{2}}{\mu^{2}}\right)-4 M_{\eta}^{2} \log \left(\frac{M_{\eta}^{2}}{\mu^{2}}\right) \\
& \left.+3\left(3 M_{\eta}^{2}+4 M_{K}^{2}+M_{\pi}^{2}\right) \log \left(\frac{M_{K}^{2}}{\mu^{2}}\right)-21 M_{\pi}^{2} \log \left(\frac{M_{\pi}^{2}}{\mu^{2}}\right)\right\} \cdot\left(\frac{\partial m_{N}}{\partial M_{\eta}}\right),
\end{aligned}
$$

and

$$
\begin{aligned}
& \sigma_{0}^{(4)}=\sigma_{\pi N}^{(4)}-\frac{1}{2 M_{\pi}}\left\{\frac{M_{\pi}^{4}\left(1152 \pi^{2}\left(2 L_{6}^{r}-L_{4}^{r}\right)-\log \left(\frac{M_{\eta}^{2}}{\mu^{2}}\right)-1\right)}{72 \pi^{2} F_{\phi}^{2}}\right\} \cdot\left(\frac{\partial m_{N}}{\partial M_{\pi}}\right) \\
& -\frac{1}{2 M_{K}}\left\{\frac { M _ { \pi } ^ { 2 } } { 2 8 8 \pi ^ { 2 } F _ { \phi } ^ { 2 } } \left[8 \left(36 \pi^{2}\left(F_{\phi}^{2}-8\left(2 L_{8}^{r}-L_{5}^{r}\right) M_{\pi}^{2}\right)\right.\right.\right. \\
& \left.+M_{K}^{2}\left(576 \pi^{2}\left(2 L_{6}^{r}-L_{4}^{r}\right)+576 \pi^{2}\left(2 L_{8}^{r}-L_{5}^{r}\right)+1\right)\right) \\
& \left.\left.+\left(9 M_{\eta}^{2}+8 M_{K}^{2}\right) \log \left(\frac{M_{\eta}^{2}}{\mu^{2}}\right)-9 M_{\pi}^{2} \log \left(\frac{M_{\pi}^{2}}{\mu^{2}}\right)\right]\right\} \cdot\left(\frac{\partial m_{N}}{\partial M_{K}}\right) \\
& -\frac{1}{2 M_{\eta}}\left\{\frac { M _ { \pi } ^ { 2 } } { 1 4 4 \pi ^ { 2 } F _ { \phi } ^ { 2 } } \left[192 \pi^{2} F_{\phi}^{2}+2304 \pi^{2}\left(2 L_{6}^{r}-L_{4}^{r}\right) M_{\eta}^{2}+4096 \pi^{2}\left(3 L_{7}^{r}+L_{8}^{r}\right) M_{K}^{2}\right.\right. \\
& -4096 \pi^{2}\left(3 L_{7}^{r}+L_{8}^{r}\right) M_{\pi}^{2}+3072 \pi^{2}\left(2 L_{8}^{r}-L_{5}^{r}\right) M_{\eta}^{2} \\
& -1536 \pi^{2}\left(2 L_{8}^{r}-L_{5}^{r}\right) M_{\pi}^{2}+5 M_{\pi}^{2}+M_{\eta}^{2}+2 M_{\pi}^{2} \log \left(\frac{M_{\eta}^{2}}{\mu^{2}}\right) \\
& -14 M_{\eta}^{2} \log \left(\frac{M_{\eta}^{2}}{\mu^{2}}\right)+3\left(3 M_{\eta}^{2}+4 M_{K}^{2}+M_{\pi}^{2}\right) \log \left(\frac{M_{K}^{2}}{\mu^{2}}\right) \\
& \left.\left.-6 M_{\pi}^{2} \log \left(\frac{M_{\pi}^{2}}{\mu^{2}}\right)\right]\right\} \cdot\left(\frac{\partial m_{N}}{\partial M_{\eta}}\right) \text {. }
\end{aligned}
$$


Using the explicit expressions for the fourth order nucleon mass in HB and covariant formulations (with and without the inclusion of the decuplet), we can calculate the derivatives of $m_{N}$ with respect to $M_{\pi}, M_{K}$ and $M_{\eta}$ to obtain the explicit expressions for the sigma-term and for $\sigma_{0}$.

\section{$5 \quad$ Fit procedure and error analysis}

We now have everything we need to calculate the quantity $\sigma_{0}$ from the nucleon mass up to fourth chiral order. But our BCHPT results for the octet-baryon masses and the sigmaterms contain LECs, whose values are unknown. So, in order to calculate $\sigma_{0}$ numerically and deduce the strangeness content of the nucleon, we have to determine the LECs first. This is achieved by fitting our BCHPT formulas to the physical values of the octet-baryon masses $N, \Sigma, \Lambda$ and $\Xi$ and to the value of the sigma-term $\sigma_{\pi N}=(59.1 \pm 3.5) \mathrm{MeV}$. Clearly, these are not sufficient data to fix all LECs. Thus, we perform two types of fits, which we call unconstrained and constrained fits, respectively, as will be discussed in what follows.

\subsection{Unconstrained fits}

We use the least-square-fitting procedure (often called $\chi^{2}$-fit) that is prominently used in CHPT to determine the LECs for various processes, see e.g. [24, 26, 30, 50]. The function $\chi^{2}\left(\lambda_{1}, \lambda_{2}, \ldots, \lambda_{n}\right)$ depends on $n$ parameters and is given by

$$
\chi^{2}\left(\lambda_{1}, \lambda_{2}, \ldots, \lambda_{n}\right)=\sum_{i=1}^{N}\left[\frac{O_{i}^{\exp }-F_{i}\left(\lambda_{1}, \lambda_{2}, \ldots, \lambda_{n}\right)}{\Delta O_{i}^{\exp }}\right]^{2},
$$

where $O_{i}^{\exp }$ are $N$ experimental observables with their respective errors $\Delta O_{i}^{\exp }$. The expression $F_{i}\left(\lambda_{1}, \lambda_{2}, \ldots, \lambda_{n}\right)$ denotes the theoretically evaluated function for the $i^{\text {th }}$ observable, which depends on the unknown parameters $\lambda_{k}$. The aim of the fit is to find the parameter values $\lambda_{k, 0}$, so that $\chi^{2}$ obtains a minimum

$$
\chi_{\min }^{2}=\min _{\lambda_{k}}\left\{\chi^{2}\left(\lambda_{1}, \ldots, \lambda_{n}\right)\right\}=\left.\chi^{2}\left(\lambda_{1}, \ldots, \lambda_{n}\right)\right|_{\left(\lambda_{1}, \ldots, \lambda_{n}\right)=\left(\lambda_{1,0}, \ldots, \lambda_{n, 0}\right)} .
$$

Then the values $\lambda_{k, 0}$ are our fit results.

We can also obtain the $n \times n$ error matrix $\mathbf{E}_{i j}$ for the fit by calculating the inverse Hessian matrix of $\chi^{2}$ evaluated at the minimum, i.e.

$$
\mathbf{E}_{i j}^{-1}=\left.\frac{1}{2} \frac{\partial^{2}}{\partial \lambda_{i} \partial \lambda_{j}}\left[\chi^{2}\left(\lambda_{1}, \ldots, \lambda_{n}\right)\right]\right|_{\left(\lambda_{1}, \ldots, \lambda_{n}\right)=\left(\lambda_{1,0}, \ldots, \lambda_{n, 0}\right)} .
$$

The error of any parameter $\lambda_{k}$ is then given by the standard deviation

$$
\Delta \lambda_{k}=\sqrt{\mathbf{E}_{k k}}
$$

and the correlation matrix is given by

$$
\mathbf{C}_{i j}=\frac{\mathbf{E}_{i j}}{\sqrt{\mathbf{E}_{i i} \mathbf{E}_{j j}}} .
$$


If we want to calculate another observable $G$, which also depends on the parameters $\lambda_{k}$, we can use the error propagation formula

$$
(\Delta G)^{2}=\left.\sum_{i, j}\left(\frac{\partial G}{\partial \lambda_{i}}\right)\left(\frac{\partial G}{\partial \lambda_{j}}\right)\right|_{\lambda_{k}=\lambda_{k, 0}} \cdot \mathbf{E}_{i j}
$$

In CHPT calculations, however, one is usually not only interested in errors from the fit, but also in theoretical errors coming from the EFT approach itself. Since the results are obtained up to a specific chiral order $\mathcal{O}\left(p^{n}\right)$, there are always corrections of $\mathcal{O}\left(p^{n+1}\right)$, that is not explicitly calculated. Thus an estimation is needed in order to see how much the result changes if higher orders are included. The truncation uncertainty of an observable $G$, which is calculated up to $\mathcal{O}\left(p^{n}\right)$, is given by $[51,52]$

$$
(\Delta G)_{\text {theo. }}^{(n)}=\max \left(\left|G^{\left(n_{\mathrm{LO}}\right)}\right| Q^{n-n_{\mathrm{LO}}+1},\left\{\left|G^{(k)}-G^{(j)}\right| Q^{n-j}\right\}\right),
$$

where $n_{\mathrm{LO}}$ denotes the order of the leading-order result with $n_{\mathrm{LO}} \leq j<k \leq n$ and $Q$ is the EFT expansion parameter given by

$$
Q=\max \left(\frac{p}{\Lambda_{\chi}}, \frac{M_{\phi}}{\Lambda_{\chi}}\right), \quad Q=\max \left(\frac{p}{\Lambda_{\chi}}, \frac{M_{\phi}}{\Lambda_{\chi}}, \frac{\Delta}{\Lambda_{\chi}}\right),
$$

with the chiral symmetry breaking scale $\Lambda_{\chi} \simeq 1 \mathrm{GeV}$, where the first (second) equation refers to the case without (with) the decuplet. In our analysis we calculate the errors from the $\chi^{2}$-fit and the theoretical errors with $Q=M_{\eta} / \Lambda_{\chi}=0.548$, which is a rather conservative estimate.

Before we perform the fits, we have to remark a last detail about the $\mathcal{O}\left(p^{4}\right)$ fit. We have a total of five experimental values $\left(m_{N}, m_{\Sigma}, m_{\Lambda}, m_{\Xi}, \sigma_{\pi N}\right)$. At order $\mathcal{O}\left(p^{2}\right)$ and $\mathcal{O}\left(p^{3}\right)$ there are four LECs $\left(m_{0}, b_{0}, b_{D}, b_{F}\right)$, which can be fitted very precisely, because we have more experimental values than LECs. However, at order $\mathcal{O}\left(p^{4}\right)$ there are 15 new LECs from meson-baryon Lagrangians entering, but we still only have 5 data to fit. In order to find at least an estimation for the $\mathcal{O}\left(p^{4}\right)$ fit, we assume that the 15 new LECs have values around zero. We therefore use a so-called prior-fit and modify our $\chi^{2}$ to, see e.g. [53],

$$
\chi_{\text {prior }}^{2}=\chi^{2}+\sum_{i=1}^{8}\left(\frac{b_{i}}{\Delta b_{i}}\right)^{2}+\sum_{i=1}^{5}\left(\frac{d_{i}}{\Delta d_{i}}\right)^{2}+\left(\frac{d_{7}}{\Delta d_{7}}\right)^{2}+\left(\frac{d_{8}}{\Delta d_{8}}\right)^{2},
$$

where $b_{i}$ are the LECs from the second order meson-baryon Lagrangian weighted by their respective errors, $\Delta b_{i}$ and $d_{i}$ are the LECs from the fourth order Lagrangian with their errors $\Delta d_{i}$. Adding these extra terms to the $\chi^{2}$, one ensures that the 15 LECs obtain values near zero (approximately Gaussian distributed), whereas the four LECs $m_{0}, b_{0}, b_{D}$ and $b_{F}$ are only fitted to the experimental data. Approximating the errors $\Delta b_{i}$ and $\Delta d_{i}$ by a constant input value, we can minimize $\chi_{\text {prior }}^{2}$ to perform the fit at $\mathcal{O}\left(p^{4}\right)$. Overall the prior-fit method behaves stable, since the minimum is not as flat as without the additional terms and all 19 LECs can be fitted quite precisely. However, we have to pay attention to choosing the errors $\Delta b_{i}$ and $\Delta d_{i}$. When the errors are too small the prior part of the fit will dominate over the actual $\chi^{2}$-fit, which is something we definitely intend to avoid. 


\subsection{Constrained fits}

In the constrained fits, the $\chi^{2}$ function for the $\mathcal{O}\left(p^{4}\right)$ case is amended by additional terms that reflect the constraints on some of the SU(3) LECs in terms of the well-known SU(2) LECs $c_{1,2,3}$ as discussed in section 3.5. As before, for the fits including the decuplet resonances, we have to subtract the $\Delta(1232)$-contribution from $c_{2}$ and $c_{3}$. We have to stress that including three new constraints from the $\mathrm{SU}(2)$ sector together with the octetbaryon masses and $\sigma_{\pi N}$ is still not a sufficient amount of experimental values to fit all 19 LECs with very good accuracy. This results in a fit, which is not as stable as the prior-fit method and does not allow a sensible error analysis. Therefore we will only present the central values of $\sigma_{0}$ obtained from this constrained fit.

\section{$6 \quad$ Results and discussion}

In our case the functions $F_{i}$ in eq. (5.1) are our calculated octet-baryon masses and the result for $\sigma_{\pi N}$ from the previous sections. The unknown parameters $\lambda_{k}$ are the LECs from the second and fourth order meson-baryon Lagrangians that we want to determine in order to calculate $\sigma_{0}$. We perform a fit for every order and for every case that we calculated, i.e. an $\mathcal{O}\left(p^{2}\right), \mathcal{O}\left(p^{3}\right)$ and $\mathcal{O}\left(p^{4}\right)$ fit for the HB approach, the HB approach with the decuplet, the covariant calculation, and the covariant calculation with the decuplet. We use the following data for the fits: the octet-baryon masses in the isospin limit $^{2}$ are $m_{N}=938.9 \mathrm{MeV}, m_{\Sigma}=1193.2 \mathrm{MeV}, m_{\Lambda}=1115.7 \mathrm{MeV}$ and $m_{\Xi}=1318.3 \mathrm{MeV}$. We use their respective standard deviations as weighting factors for the $\chi^{2}$-fit. The standard deviation of the nucleon mass, for example, is given by

$$
\left(\Delta m_{N}\right):=\left[\frac{\left(m_{\text {proton }}-m_{N}\right)^{2}+\left(m_{\text {neutron }}-m_{N}\right)^{2}}{2}\right]^{\frac{1}{2}},
$$

with the PDG values for the proton and neutron mass. We obtain the standard deviations $\Delta m_{N}=0.65 \mathrm{MeV}, \Delta m_{\Sigma}=3.30 \mathrm{MeV}, \Delta m_{\Xi}=3.40 \mathrm{MeV}$, which will be used as our experimental uncertainties. Since there is only one $\Lambda$-baryon, we assume $\Delta m_{\Lambda}=1.00 \mathrm{MeV}$. As already mentioned, we use $\sigma_{\pi N}=(59.1 \pm 3.5) \mathrm{MeV}$ for $\sigma_{\pi N}$ and its uncertainty. The meson masses are $M_{\pi}=139 \mathrm{MeV}$ and $M_{K}=494 \mathrm{MeV}$. Up to $\mathcal{O}\left(p^{3}\right)$ we use the Gell-MannOkubo relation to obtain the $\eta$ mass, but at $\mathcal{O}\left(p^{4}\right)$ we use the PDG value $M_{\eta}=548 \mathrm{MeV}$. The other constants are given by

$$
F_{\phi}=1.17 \cdot F_{\pi} \simeq 108 \mathrm{MeV}, \quad D=0.80, \quad F=0.46,
$$

$F_{\phi}$ the average of the pion, the kaon and the eta decay constants. Inside the loop functions we set the average octet-baryon mass in the chiral limit $m_{0}$ equal to $m_{B}=1.151 \mathrm{GeV}$ (which is the average octet mass) and, analogously, the decuplet mass to $m_{D}=1.382 \mathrm{GeV}$ (which is the average decuplet mass). The average mass splitting between the octet and the decuplet, $\Delta$, is then given by $\Delta=m_{D}-m_{B}=0.231 \mathrm{GeV}$. For the octet-decuplet-meson coupling $\mathcal{C}$, we use $\mathcal{C}=1.7$ and the off-shell parameter $z$ is set to $z=1 / 2$ in the covariant

\footnotetext{
${ }^{2}$ For a given multiplet, this is nothing but the sum of the masses divided by the number of states.
} 


\begin{tabular}{|c|c|c|c|c|}
\hline & HB & HB + decuplet & EOMS & EOMS + decuplet \\
\hline $\mathcal{O}\left(p^{3}\right)$ & $57.9(0.2)(17.0)$ & $88.6(0.2)(34.0)$ & $46.4(0.2)(10.4)$ & $57.6(0.2)(17.0)$ \\
$\mathcal{O}\left(p^{4}\right)$ & $64.1(31.7)(9.3)$ & $64.0(31.7)(18.7)$ & $51.8(31.4)(5.7)$ & $61.8(31.4)(9.3)$ \\
\hline
\end{tabular}

Table 7. Results for $\sigma_{0}$ in $\mathrm{MeV}$ at third and fourth order. Here, "+decuplet" means the inclusion of the decuplet, HB denotes the heavy baryon and EOMS the covariant approach. The first error comes from the uncertainties within the given order, the second error is an estimate of the neglected higher order effects based on eq. (5.7).

calculation. The LECs $L_{4}, L_{5}, L_{6}, L_{7}, L_{8}$ from the fourth order meson Lagrangian and $t_{0}, t_{D}$ from the second order decuplet Lagrangian, which enter the baryon mass formulas at $\mathcal{O}\left(p^{4}\right)$, have been obtained from other sources. The values for the former are from ref. [54], while the results for the latter are given in appendix B. Throughout, we use the renormalization scale $\mu=m_{D}=1.382 \mathrm{GeV}$.

The fit results for the LECs can be found in appendix $\mathrm{C}$ for one example, namely the covariant calculation without the decuplet at $\mathcal{O}\left(p^{2}\right), \mathcal{O}\left(p^{3}\right)$ and $\mathcal{O}\left(p^{4}\right)$. For the prior-fit we used the errors $\Delta b_{1, \ldots, 4}=0.1 \mathrm{GeV}^{-1}, \Delta b_{5, \ldots, 8}=0.1 \mathrm{GeV}^{-2}$ and $\Delta d_{1, \ldots, 5,7,8}=0.1 \mathrm{GeV}^{-3}$. We note that the choice of larger uncertainties leads to a larger error. With these values we calculate the quantity $\sigma_{0}$ and its errors using the error propagation formula, eq. (5.6) and the theoretical truncation error estimation, eq. (5.7). With the results from the $\mathcal{O}\left(p^{2}\right)$ fit, we obtain

$$
\sigma_{0}=27.4(0.2)(14.8) \mathrm{MeV},
$$

where the first bracket gives the uncertainty from the fit and the second gives the theoretical uncertainty. The results for the higher chiral orders are given in table 7. Similar results for the $\mathcal{O}\left(p^{3}\right)$ fit have been obtained earlier in [13]. The $\mathcal{O}\left(p^{4}\right)$ results for the covariant calculation only differ slightly from the $\mathcal{O}\left(p^{3}\right)$ results. The fit without decuplet-resonances shifts more towards $\sigma_{\pi N}$, while the fit with the decuplet is slightly above $60 \mathrm{MeV}$. The $\mathcal{O}\left(p^{4}\right)$ HB fit results are both around $64 \mathrm{MeV}$. So the $\mathcal{O}\left(p^{4}\right)$ result for the HB approach including decuplet baryons is much closer to $\sigma_{\pi N}$ than its corresponding $\mathcal{O}\left(p^{3}\right)$ result. This supports the idea that there are higher order terms contributing significantly, which are not contained in the $\mathcal{O}\left(p^{3}\right)$ calculation.

Considering the errors of the calculation, we see that the errors from the fit are quite small for the $\mathcal{O}\left(p^{3}\right)$ calculations. This is due to the fact that we have more experimental data than LECs and thus the fit behaves well. The errors of the $\mathcal{O}\left(p^{4}\right)$ calculations are very large because of the prior-fit method that we use. Since we assume that all 15 LECs from the $\mathcal{O}\left(p^{4}\right)$ calculation have the same error, we are not able to include any correlations between them, which might reduce the error of $\sigma_{0}$.

The theoretical error due to cutting off higher orders decreases overall from $\mathcal{O}\left(p^{3}\right)$ to $\mathcal{O}\left(p^{4}\right)$, as one would expect. The error from $\mathcal{O}\left(p^{2}\right)$, however, is slightly smaller than most errors from $\mathcal{O}\left(p^{3}\right)$. The reason for this is that all $\mathcal{O}\left(p^{3}\right)$ results (besides the covariant result without the decuplet) are close to the $\sigma_{\pi N}$ value and differ by roughly $30 \mathrm{MeV}$ from the 


\begin{tabular}{|c|c|c|c|c|}
\hline & HB & HB + decuplet & EOMS & EOMS + decuplet \\
\hline$\sigma_{0}[\mathrm{MeV}]$ & 58.0 & 60.4 & 42.8 & 60.9 \\
\hline
\end{tabular}

Table 8. Values of $\sigma_{0}$ for the $\mathcal{O}\left(p^{4}\right)$ constrained fits.

$\mathcal{O}\left(p^{2}\right)$ tree-level result. This implies that the loop contribution plays an important role in the calculation of $\sigma_{0}$.

Given the central values for $\sigma_{0}$ from table 7 , we see that the strangeness content $y \simeq 0$, but due to the large uncertainties, this can not be made more precise.

Consider now the constraint fit as described above. As already mentioned, we only give the central values for $\sigma_{0}$ here. For $c_{1,2,3}$ we use their numerical values given in section 3.5 and their respective errors as weighting factors for the fit. We also use $\bar{e}_{1}=-1 \mathrm{GeV}^{-3}$. Fits including the decuplet resonances are modified by subtracting the delta contribution from $c_{2}$ and $c_{3}$. Since the error of the $\Delta(1232)$-contribution is assumed to be quite large, see [27], we adjust the errors of $\left(c_{2}-c_{2}^{\Delta}\right)$ and $\left(c_{3}-c_{3}^{\Delta}\right)$ to $\pm 1.0 \mathrm{GeV}^{-1}$. The constant $c_{1}$ remains unchanged.

Overall we see that the value for $\sigma_{0}$ drops in comparison to the prior-fit results, cf. table 8. This also supports the idea of a vanishing strangeness content.

\section{Conclusions and outlook}

In this paper, we have analyzed the ground-state octet baryon masses, the pion-nucleon $\sigma$-term and its flavor decomposition to fourth order in the chiral expansion, using the heavy baryon and the covariant EOMS approach as well as including the contributions from the low-lying baryon decuplet. We have entirely relied on continuum data and phenomenology to fix the pertinent LECs. Here, we have mainly focused on the prediction for $\sigma_{0}$, always using the value of $\sigma_{\pi N}=(59.1 \pm 3.5) \mathrm{MeV}$ from the Roy-Steiner analysis of pion-nucleon scattering. The main findings of our investigation are:

1. At third order, there is a large spread in the results for $\sigma_{0}$ depending on the BCHPT scheme and whether or not the decuplet is included, cf. table 7 . The uncertainties from the LECs within this order are very small. This confirms earlier findings of ref. [13]. In addition, we have shown that the error due to the neglect of higher orders is large.

2. At fourth order, the central values of the fits are much closer, ranging from $52 \mathrm{MeV}$ to $64 \mathrm{MeV}$, consistent with a small strangeness fraction $y \simeq 0$. However, since not all LECs can be determined and the fit must be supplemented by a Bayesian ansatz for some of the LECs, the fit error within the order is sizeable, as expected. The uncertainty from neglecting higher orders is, however, much reduced compared to the third order.

3. Constraining certain combinations of the $\mathrm{SU}(3)$ LECs from matching to the $\mathrm{SU}(2)$ LECs, that are known to high precision from the Roy-Steiner analysis of pion-nucleon scattering, leads to a downward shift of a few $\mathrm{MeV}$ in the central value of $\sigma_{0}$. 
Once the apparent discrepancy in the determination of $\sigma_{\pi N}$ from Roy-Steiner equations and the lattice is resolved, the formalism developed here can also be used to analyze lattice results at varying quark masses. This should give a better handle on the badly determined LECs.

\section{Acknowledgments}

We would like to thank Akaki Rusetsky for useful discussions. Work supported in part by funds provided from the Deutsche Forschungsgemeinschaft (SFB/TRR 110, "Symmetries and the Emergence of Structure in QCD"), by the Chinese Academy of Sciences (CAS) President's International Fellowship Initiative (PIFI) (grant no. 2018DM0034) and by VolkswagenStiftung (grant no. 93562).

\section{A Mesonic chiral Lagrangian}

For completeness, we discuss here the LO and NLO chiral Lagrangian of the Goldstone boson fields. The LO Lagrangian is given by $[48,55]$,

$$
\mathcal{L}_{\phi}^{(2)}=\frac{F_{\phi}^{2}}{4} \operatorname{Tr}\left(D_{\mu} U\left(D^{\mu} U\right)^{\dagger}\right)+\frac{F_{\phi}^{2}}{4} \operatorname{Tr}\left(\chi U^{\dagger}+U \chi^{\dagger}\right) .
$$

Here, $F_{\phi}$ is the pseudoscalar decay constant in the chiral limit and the trace acts in flavor space. The pseudoscalar fields are written in terms of a unitary $3 \times 3$ matrix $U$, which is defined by

$$
U(x)=\exp \left(i \frac{\phi(x)}{F_{\phi}}\right)=\mathbb{1}+i \frac{\phi(x)}{F_{\phi}}-\frac{\phi^{2}(x)}{2 F_{\phi}^{2}}+\ldots,
$$

with

$$
\phi(x)=\sum_{a=1}^{8} \lambda^{a} \phi^{a}(x)=\sqrt{2}\left(\begin{array}{cccc}
\frac{1}{\sqrt{2}} \pi^{0}+\frac{1}{\sqrt{6}} \eta & \pi^{+} & K^{+} \\
\pi^{-} & -\frac{1}{\sqrt{2}} \pi^{0}+\frac{1}{\sqrt{6}} \eta & K^{0} \\
K^{-} & \bar{K}^{0} & -\frac{2}{\sqrt{6}} \eta
\end{array}\right) .
$$

The matrix $U$ transforms as $U \rightarrow R U L^{\dagger}$ under global $\mathrm{SU}(3)_{L} \times \mathrm{SU}(3)_{R}$ transformations $\left(R, L \in \mathrm{SU}(3)_{R, L}\right) . D_{\mu}$ is the covariant derivative defined by

$$
D_{\mu} U:=\partial_{\mu} U-i r_{\mu} U+i U l_{\mu}
$$

with $r_{\mu}=v_{\mu}+a_{\mu}$ and $l_{\mu}=v_{\mu}-a_{\mu}$, where $v_{\mu}$ and $a_{\mu}$ denote external vector and axial vector currents, respectively. ${ }^{3}$ The covariant derivative transforms as $D_{\mu} U \rightarrow R\left(D_{\mu} U\right) L^{\dagger}$. The second term in (A.1) includes the explicit chiral symmetry breaking due to the non-zero quark masses

$$
\chi=2 B_{0} \mathcal{M},
$$

where $B_{0}$ is a constant related to the chiral quark condensate and $\mathcal{M}=\operatorname{diag}\left(m_{u}, m_{d}, m_{s}\right)$ is the quark mass matrix transforming as $\mathcal{M} \rightarrow R \mathcal{M} L^{\dagger}$.

\footnotetext{
${ }^{3}$ We are only interested in strong interaction processes and therefore we set $v_{\mu}=a_{\mu}=0$ in the following.
} 
At order $\mathcal{O}\left(p^{4}\right)(\mathrm{NLO})$ there are new terms that contribute to the meson Lagrangian

$$
\begin{aligned}
\mathcal{L}_{\phi}^{(4)}= & L_{1}\left[\operatorname{Tr}\left(D_{\mu} U\left(D^{\mu} U\right)^{\dagger}\right)\right]^{2}+L_{2} \operatorname{Tr}\left(D_{\mu} U\left(D_{\nu} U\right)^{\dagger}\right) \operatorname{Tr}\left(D^{\mu} U\left(D^{\nu} U\right)^{\dagger}\right) \\
& +L_{3} \operatorname{Tr}\left(D_{\mu} U\left(D^{\mu} U\right)^{\dagger} D_{\nu} U\left(D^{\nu} U\right)^{\dagger}\right)+L_{4} \operatorname{Tr}\left(D_{\mu} U\left(D^{\mu} U\right)^{\dagger}\right) \operatorname{Tr}\left(\chi U^{\dagger}+U \chi^{\dagger}\right) \\
& +L_{5} \operatorname{Tr}\left(D_{\mu} U\left(D^{\mu} U\right)^{\dagger}\left(\chi U^{\dagger}+U \chi^{\dagger}\right)\right)+L_{6}\left[\operatorname{Tr}\left(\chi U^{\dagger}+U \chi^{\dagger}\right)\right]^{2} \\
& +L_{7}\left[\operatorname{Tr}\left(\chi U^{\dagger}-U \chi^{\dagger}\right)\right]^{2}+L_{8} \operatorname{Tr}\left(\chi U^{\dagger} \chi U^{\dagger}+U \chi^{\dagger} U \chi^{\dagger}\right)+\ldots,
\end{aligned}
$$

where $L_{1}, L_{2}, \ldots, L_{7}$, and $L_{8}$ are LECs. The other terms of the $\mathcal{O}\left(p^{4}\right)$ Lagrangian, that are not listed in eq. (A.6) contain external vector and axial vector currents and thus are not of relevance to our calculation. There are also two terms without meson fields proportional to the high-energy constants (HECs) $H_{1}$ and $H_{2}$. But they also do not contribute to our calculation.

\section{B Decuplet-baryon masses at $\mathcal{O}\left(p^{2}\right)$}

The decuplet-baryon masses at $\mathcal{O}\left(p^{2}\right)$ can be calculated from the chiral symmetry breaking Lagrangian in eq. (3.19). They are given by [56]

$$
\begin{aligned}
& m_{\Delta}^{(2)}=-\left(t_{0}+3 t_{D}\right) M_{\pi}^{2}-2 t_{0} M_{K}^{2}, \\
& m_{\Sigma^{*}}^{(2)}=-\left(t_{0}+t_{D}\right) M_{\pi}^{2}-\left(2 t_{0}+2 t_{D}\right) M_{K}^{2}, \\
& m_{\Xi^{*}}^{(2)}=-\left(t_{0}-t_{D}\right) M_{\pi}^{2}-\left(2 t_{0}+4 t_{D}\right) M_{K}^{2}, \\
& m_{\Omega^{-}}^{(2)}=-\left(t_{0}-3 t_{D}\right) M_{\pi}^{2}-\left(2 t_{0}+6 t_{D}\right) M_{K}^{2} .
\end{aligned}
$$

The LECs $t_{0}$ and $t_{D}$ can be determined from a fit to the $\mathcal{O}\left(p^{3}\right)$ decuplet-baryon masses. Their values are $t_{0}=-0.27 \mathrm{GeV}^{-1}$ and $t_{D}=-0.694 \mathrm{GeV}^{-1}$ (assuming an average decuplet mass of $m_{D}=1.382 \mathrm{GeV}$ in the chiral limit) [56].

\section{Fit results}

As one representative, we exhibit the values of the LECs for the covariant calculation without the decuplet in table 9. The other fit results are given in ref. [49]. 


\begin{tabular}{|l|c|c|c|}
\hline & Fit $\mathcal{O}\left(p^{2}\right)$ & Fit $\mathcal{O}\left(p^{3}\right)$ & Fit $\mathcal{O}\left(p^{4}\right)$ \\
\hline$m_{0}[\mathrm{MeV}]$ & $(650.0 \pm 46.0)$ & $(660.5 \pm 44.7)$ & $(770.0 \pm 56.7)$ \\
$b_{0}\left[\mathrm{GeV}^{-1}\right]$ & $(-0.536 \pm 0.045)$ & $(-0.873 \pm 0.045)$ & $(-0.542 \pm 0.036)$ \\
$b_{D}\left[\mathrm{GeV}^{-1}\right]$ & $(0.063 \pm 0.003)$ & $(0.063 \pm 0.003)$ & $(0.081 \pm 0.020)$ \\
$b_{F}\left[\mathrm{GeV}^{-1}\right]$ & $(-0.216 \pm 0.001)$ & $(-0.422 \pm 0.001)$ & $(-0.313 \pm 0.003)$ \\
$b_{1}\left[\mathrm{GeV}^{-1}\right]$ & - & - & $(-0.001 \pm 0.100)$ \\
$b_{2}\left[\mathrm{GeV}^{-1}\right]$ & - & - & $(-0.001 \pm 0.100)$ \\
$b_{3}\left[\mathrm{GeV}^{-1}\right]$ & - & - & $(-0.013 \pm 0.100)$ \\
$b_{4}\left[\mathrm{GeV}^{-1}\right]$ & - & - & $(-0.003 \pm 0.100)$ \\
$b_{5}\left[\mathrm{GeV}^{-2}\right]$ & - & - & $(0.000 \pm 0.100)$ \\
$b_{6}\left[\mathrm{GeV}^{-2}\right]$ & - & - & $(-0.007 \pm 0.100)$ \\
$b_{7}\left[\mathrm{GeV}^{-2}\right]$ & - & - & $(0.000 \pm 0.100)$ \\
$b_{8}\left[\mathrm{GeV}^{-2}\right]$ & - & - & $(-0.001 \pm 0.100)$ \\
$d_{1}\left[\mathrm{GeV}^{-3}\right]$ & - & - & $(0.034 \pm 0.100)$ \\
$d_{2}\left[\mathrm{GeV}^{-3}\right]$ & - & - & $(0.016 \pm 0.100)$ \\
$d_{3}\left[\mathrm{GeV}^{-3}\right]$ & - & - & $(0.074 \pm 0.100)$ \\
$d_{4}\left[\mathrm{GeV}^{-3}\right]$ & - & $-0.055 \pm 0.100)$ \\
$d_{5}\left[\mathrm{GeV}^{-3}\right]$ & - & - & $(0.071 \pm 0.100)$ \\
$d_{7}\left[\mathrm{GeV}^{-3}\right]$ & - & - & $(0.056 \pm 0.100)$ \\
$d_{8}\left[\mathrm{GeV}^{-3}\right]$ & - & - & \\
\hline
\end{tabular}

Table 9. Fitted LECs are second, third and fourth order for the covariant calculation without the decuplet as decribed in the text.

Open Access. This article is distributed under the terms of the Creative Commons Attribution License (CC-BY 4.0), which permits any use, distribution and reproduction in any medium, provided the original author(s) and source are credited.

\section{References}

[1] A. Bottino, F. Donato, N. Fornengo and S. Scopel, Implications for relic neutralinos of the theoretical uncertainties in the neutralino nucleon cross-section, Astropart. Phys. 13 (2000) 215 [hep-ph/9909228] [INSPIRE].

[2] J.R. Ellis, K.A. Olive and C. Savage, Hadronic uncertainties in the elastic scattering of supersymmetric dark matter, Phys. Rev. D 77 (2008) 065026 [arXiv:0801.3656] [INSPIRE].

[3] A. Crivellin, M. Hoferichter and M. Procura, Accurate evaluation of hadronic uncertainties in spin-independent WIMP-nucleon scattering: disentangling two- and three-flavor effects, Phys. Rev. D 89 (2014) 054021 [arXiv: 1312.4951] [InSPIRE]. 
[4] A. Crivellin, M. Hoferichter and M. Procura, Improved predictions for $\mu \rightarrow e$ conversion in nuclei and Higgs-induced lepton flavor violation, Phys. Rev. D 89 (2014) 093024 [arXiv: 1404.7134] [INSPIRE].

[5] J. de Vries, E. Mereghetti, C.-Y. Seng and A. Walker-Loud, Lattice QCD spectroscopy for hadronic CP-violation, Phys. Lett. B 766 (2017) 254 [arXiv: 1612.01567] [INSPIRE].

[6] J.F. Donoghue, E. Golowich and B.R. Holstein, Dynamics of the Standard Model, Camb. Monogr. Part. Phys. Nucl. Phys. Cosmol. 2 (1992) 1 [Camb. Monogr. Part. Phys. Nucl. Phys. Cosmol. 35 (2014) 1] [InSPIRE].

[7] B. Borasoy and U.-G. Meißner, Chiral expansion of baryon masses and $\sigma$-terms, Annals Phys. 254 (1997) 192 [hep-ph/9607432] [INSPIRE].

[8] J. Gasser, H. Leutwyler and M.E. Sainio, Sigma term update, Phys. Lett. B 253 (1991) 252 [INSPIRE].

[9] M. Hoferichter, J. Ruiz de Elvira, B. Kubis and U.-G. Meißner, High-precision determination of the pion-nucleon $\sigma$-term from Roy-Steiner equations, Phys. Rev. Lett. 115 (2015) 092301 [arXiv: 1506.04142] [INSPIRE].

[10] M. Hoferichter, J. Ruiz de Elvira, B. Kubis and U.-G. Meißner, Roy-Steiner-equation analysis of pion-nucleon scattering, Phys. Rept. 625 (2016) 1 [arXiv:1510.06039] [INSPIRE].

[11] J. Ruiz de Elvira, M. Hoferichter, B. Kubis and U.-G. Meißner, Extracting the $\sigma$-term from low-energy pion-nucleon scattering, J. Phys. G 45 (2018) 024001 [arXiv:1706.01465] [INSPIRE].

[12] J. Gasser, Hadron masses and sigma commutator in the light of chiral perturbation theory, Annals Phys. 136 (1981) 62 [INSPIRE].

[13] J.M. Alarcon, L.S. Geng, J. Martin Camalich and J.A. Oller, The strangeness content of the nucleon from effective field theory and phenomenology, Phys. Lett. B 730 (2014) 342 [arXiv:1209.2870] [INSPIRE].

[14] A. Krause, Baryon matrix elements of the vector current in chiral perturbation theory, Helv. Phys. Acta 63 (1990) 3 [INSPIRE].

[15] M. Frink and U.-G. Meißner, On the chiral effective meson-baryon Lagrangian at third order, Eur. Phys. J. A 29 (2006) 255 [hep-ph/0609256] [INSPIRE].

[16] J.A. Oller, M. Verbeni and J. Prades, Meson-baryon effective chiral lagrangians to $O\left(q^{3}\right)$, JHEP 09 (2006) 079 [hep-ph/0608204] [INSPIRE].

[17] X.-L. Ren, L.S. Geng, J. Martin Camalich, J. Meng and H. Toki, Octet baryon masses in next-to-next-to-next-to-leading order covariant baryon chiral perturbation theory, JHEP 12 (2012) 073 [arXiv: 1209.3641] [INSPIRE].

[18] J. Gasser, M.E. Sainio and A. Svarc, Nucleons with chiral loops, Nucl. Phys. B 307 (1988) 779 [INSPIRE].

[19] E.E. Jenkins and A.V. Manohar, Baryon chiral perturbation theory using a heavy fermion Lagrangian, Phys. Lett. B 255 (1991) 558 [INSPIRE].

[20] V. Bernard, N. Kaiser, J. Kambor and U.-G. Meißner, Chiral structure of the nucleon, Nucl. Phys. B 388 (1992) 315 [INSPIRE].

[21] V. Bernard, N. Kaiser and U.-G. Meißner, Chiral dynamics in nucleons and nuclei, Int. J. Mod. Phys. E 4 (1995) 193 [hep-ph/9501384] [InSPIRE]. 
[22] V. Bernard, Chiral perturbation theory and baryon properties, Prog. Part. Nucl. Phys. 60 (2008) 82 [arXiv: 0706.0312] [inSPIRE].

[23] T. Fuchs, J. Gegelia, G. Japaridze and S. Scherer, Renormalization of relativistic baryon chiral perturbation theory and power counting, Phys. Rev. D 68 (2003) 056005 [hep-ph/0302117] [INSPIRE].

[24] D. Siemens, V. Bernard, E. Epelbaum, A. Gasparyan, H. Krebs and U.-G. Meißner, Elastic pion-nucleon scattering in chiral perturbation theory: a fresh look, Phys. Rev. C 94 (2016) 014620 [arXiv: 1602.02640] [INSPIRE].

[25] E.E. Jenkins and A.V. Manohar, Chiral corrections to the baryon axial currents, Phys. Lett. B 259 (1991) 353 [INSPIRE].

[26] V. Bernard, N. Kaiser and U.-G. Meißner, Critical analysis of baryon masses and $\sigma$ terms in heavy baryon chiral perturbation theory, Z. Phys. C 60 (1993) 111 [hep-ph/9303311] [INSPIRE].

[27] V. Bernard, N. Kaiser and U.-G. Meißner, Aspects of chiral pion-nucleon physics, Nucl. Phys. A 615 (1997) 483 [hep-ph/9611253] [INSPIRE].

[28] H. Krebs, E. Epelbaum and U.-G. Meißner, Redundancy of the off-shell parameters in chiral effective field theory with explicit spin-3/2 degrees of freedom, Phys. Lett. B 683 (2010) 222 [arXiv: 0905.2744] [INSPIRE].

[29] H.-B. Tang and P.J. Ellis, Redundance of $\Delta$-isobar parameters in effective field theories, Phys. Lett. B 387 (1996) 9 [hep-ph/9606432] [INSPIRE].

[30] D.-L. Yao et al., Pion-nucleon scattering in covariant baryon chiral perturbation theory with explicit Delta resonances, JHEP 05 (2016) 038 [arXiv: 1603.03638] [INSPIRE].

[31] N. Wies, J. Gegelia and S. Scherer, Consistency of the $\pi \Delta$ interaction in chiral perturbation theory, Phys. Rev. D 73 (2006) 094012 [hep-ph/0602073] [INSPIRE].

[32] T.R. Hemmert, B.R. Holstein and J. Kambor, Chiral Lagrangians and $\Delta(1232)$ interactions: formalism, J. Phys. G 24 (1998) 1831 [hep-ph/9712496] [INSPIRE].

[33] R.D. Young, D.B. Leinweber and A.W. Thomas, Convergence of chiral effective field theory, Prog. Part. Nucl. Phys. 50 (2003) 399 [hep-lat/0212031] [InSPIRE].

[34] V. Bernard, T.R. Hemmert and U.-G. Meißner, Cutoff schemes in chiral perturbation theory and the quark mass expansion of the nucleon mass, Nucl. Phys. A 732 (2004) 149 [hep-ph/0307115] [INSPIRE].

[35] M. Procura, T.R. Hemmert and W. Weise, Nucleon mass, $\sigma$ term and lattice QCD, Phys. Rev. D 69 (2004) 034505 [hep-lat/0309020] [INSPIRE].

[36] S.R. Beane, In search of the chiral regime, Nucl. Phys. B 695 (2004) 192 [hep-lat/0403030] [INSPIRE].

[37] M. Frink, U.-G. Meißner and I. Scheller, Baryon masses, chiral extrapolations and all that, Eur. Phys. J. A 24 (2005) 395 [hep-lat/0501024] [InSPIRE].

[38] A. Walker-Loud, Evidence for non-analytic light quark mass dependence in the baryon spectrum, Phys. Rev. D 86 (2012) 074509 [arXiv:1112.2658] [INSPIRE].

[39] A. Semke and M.F.M. Lutz, Strangeness in the baryon ground states, Phys. Lett. B 717 (2012) 242 [arXiv:1202.3556] [INSPIRE]. 
[40] M.F.M. Lutz, Y. Heo and X.-Y. Guo, On the convergence of the chiral expansion for the baryon ground-state masses, Nucl. Phys. A 977 (2018) 146 [arXiv:1801.06417] [InSPIRE].

[41] M. Hoferichter, J. Ruiz de Elvira, B. Kubis and U.-G. Meißner, Remarks on the pion-nucleon $\sigma$-term, Phys. Lett. B 760 (2016) 74 [arXiv: 1602.07688] [inSPIRE].

[42] M. Frink and U.-G. Meißner, Chiral extrapolations of baryon masses for unquenched three flavor lattice simulations, JHEP 07 (2004) 028 [hep-lat/0404018] [INSPIRE].

[43] D. Siemens et al., Reconciling threshold and subthreshold expansions for pion-nucleon scattering, Phys. Lett. B 770 (2017) 27 [arXiv:1610.08978] [INSPIRE].

[44] M. Mai, P.C. Bruns, B. Kubis and U.-G. Meißner, Aspects of meson-baryon scattering in three and two-flavor chiral perturbation theory, Phys. Rev. D 80 (2009) 094006 [arXiv:0905.2810] [INSPIRE].

[45] M. Hoferichter, J. Ruiz de Elvira, B. Kubis and U.-G. Meißner, Matching pion-nucleon Roy-Steiner equations to chiral perturbation theory, Phys. Rev. Lett. 115 (2015) 192301 [arXiv: 1507.07552] [INSPIRE].

[46] J.A. Oller, On the strangeness -1 S-wave meson-baryon scattering, Eur. Phys. J. A 28 (2006) 63 [hep-ph/0603134] [INSPIRE].

[47] B.C. Lehnhart, J. Gegelia and S. Scherer, Baryon masses and nucleon sigma terms in manifestly Lorentz-invariant baryon chiral perturbation theory, J. Phys. G 31 (2005) 89 [hep-ph/0412092] [INSPIRE].

[48] J. Gasser and H. Leutwyler, Chiral perturbation theory: expansions in the mass of the strange quark, Nucl. Phys. B 250 (1985) 465 [INSPIRE].

[49] D. Severt, Sigma-term physics, Master's thesis, University of Bonn, Bonn, Germany (2018).

[50] R. Navarro Perez, J.E. Amaro and E. Ruiz Arriola, Statistical error analysis for phenomenological nucleon-nucleon potentials, Phys. Rev. C 89 (2014) 064006 [arXiv: 1404.0314] [INSPIRE].

[51] E. Epelbaum, H. Krebs and U.-G. Meißner, Improved chiral nucleon-nucleon potential up to next-to-next-to-next-to-leading order, Eur. Phys. J. A 51 (2015) 53 [arXiv:1412.0142] [INSPIRE].

[52] LENPIC collaboration, Few-nucleon systems with state-of-the-art chiral nucleon-nucleon forces, Phys. Rev. C 93 (2016) 044002 [arXiv:1505.07218] [INSPIRE].

[53] M.R. Schindler and D.R. Phillips, Bayesian methods for parameter estimation in effective field theories, Annals Phys. 324 (2009) 682 [Erratum ibid. 324 (2009) 2051] [arXiv:0808.3643] [INSPIRE].

[54] J. Bijnens and I. Jemos, A new global fit of the $L_{i}^{r}$ at next-to-next-to-leading order in chiral perturbation theory, Nucl. Phys. B 854 (2012) 631 [arXiv:1103.5945] [INSPIRE].

[55] J. Gasser and H. Leutwyler, Chiral perturbation theory to one loop, Annals Phys. 158 (1984) 142 [INSPIRE].

[56] J. Martin Camalich, L.S. Geng and M.J. Vicente Vacas, The lowest-lying baryon masses in covariant SU(3)-flavor chiral perturbation theory, Phys. Rev. D 82 (2010) 074504 [arXiv: 1003.1929] [INSPIRE]. 